\title{
Rapidly Decaying Solutions of the Nonlinear Schrödinger Equation
}

\section{Thierry Cazenave ${ }^{1}$ and Fred B. Weissler ${ }^{2,3}$}

1 Analyse Numérique, Université Pierre et Marie Curie, 4, place Jussieu, F-75252 Paris Cedex 05, France

2 Centre de Mathématiques, ENS Cachan, 61, Avenue du Président Wilson, F-94235 Cachan Cedex, France

${ }^{3}$ UFR de Sciences, Université Paris XII, 61, Avenue du Général de Gaulle, F-94010 Créteil Cedex, France

Received April 8, 1991

Abstract. We consider global solutions of the nonlinear Schrödinger equation

$$
i u_{t}+\Delta u=\lambda|u|^{\alpha} u, \quad \text { in } \mathbf{R}^{N},
$$

where $\lambda \in \mathbf{R}$ and $0<\alpha<\frac{4}{N-2}$. In particular, for $\alpha>\alpha_{0}=\frac{2-N+\sqrt{N^{2}+12 N+4}}{2 N}$, we show that for every $\varphi \in H^{1}\left(\mathbf{R}^{N}\right)$ such that $x \varphi(x) \in L^{2}\left(\mathbf{R}^{N}\right)$, the solution of (NLS) with initial value $\varphi(x) e^{i\left(b|x|^{2} / 4\right)}$ is global and rapidly decaying as $t \rightarrow \infty$ if $b$ is large enough. Furthermore, by applying the pseudo-conformal transformation and studying the resulting nonautonomous nonlinear Schrödinger equation, we obtain both new results and simpler proofs of some known results concerning the scattering theory. In particular, we construct the wave operators for $\frac{4}{N+2}<\alpha<\frac{4}{N-2}$. Also, we establish a low energy scattering theory for the same range of $\alpha$ and show that, at least for $\lambda<0$, the lower bound on $\alpha$ is optimal. Finally; if $\lambda>0$, we prove asymptotic completeness for $\alpha_{0} \leqq \alpha<\frac{4}{N-2}$.

\section{Introduction}

In this paper we study solutions in $\mathbf{R}^{N}$ of the nonlinear Schrödinger equation

$$
i u_{t}+\Delta u=\lambda|u|^{\alpha} u \text {. }
$$

Here $u=u(t, x)$ is a complex valued function defined for $t$ in some subset of the real numbers and all $x \in \mathbf{R}^{N}, \lambda \in \mathbf{R}$, and $0<\alpha<\frac{4}{N-2}$. We frequently write $u(t)$ for the 
spatial function $u(t, \cdot)$. One natural point of view in studying solutions of (1.1) is to study the associated Cauchy problem, where the initial value $\varphi=u(0)$ is specified. As is well known (see for example $[18,4]$ ), this Cauchy problem is formally equivalent to the integral equation

$$
u(t)=S(t) u(0)-i \lambda \int_{0}^{t} S(t-s)|u|^{\alpha} u(s) d s,
$$

where

$$
S(t)=e^{i t \Delta}
$$

is the unitary group determined by the linear Schrödinger equation, i.e. when $\lambda=0$. $S(t)$ is given by the well-known complex Gauss kernel,

$$
S(t) \varphi(x)=(4 \pi i t)^{-N / 2} \int_{\mathbf{R}^{N}} e^{i\left(|x-y|^{2} / 4 t\right)} \varphi(y) d y .
$$

We are primarily concerned with (positively) global solutions to (1.1), that is solutions which are defined for all $t \geqq 0$; and our goal is to study the decay and scattering properties of such solutions. In particular, we define below a solution $u$ to be rapidly decaying if a certain space-time integral of $u$ is finite; and we give (for $\alpha$ in a certain range) a sufficient condition on the initial data $\varphi$ for $u$ to have rapid decay. In the case $\lambda<0$ and $\alpha \geqq 4 / N$, this yields a new global existence result and gives some insight into finite time blow up. Also, we improve (on the lower side) the range of $\alpha$ for which certain scattering properties of solutions to (1.1) are known.

Before describing our results in more detail, we recall some basic facts about solutions to (1.1) and (1.2). For $0<\alpha<\frac{4}{N-2}$ the Cauchy problem corresponding to (1.1) is well posed both in $H^{1}\left(\mathbf{R}^{N}\right)$ and in the space

$$
X=\left\{u \in H^{1}\left(\mathbf{R}^{N}\right) ;|\cdot| u(\cdot) \in L^{2}\left(\mathbf{R}^{N}\right)\right\} .
$$

One first proves local existence and uniqueness of solutions to (1.2), and then shows that the solution satisfies (1.1) in an appropriate sense. For precise statements see $[10,4]$. Moreover, these solutions satisfy the following conservation laws:

$$
\begin{array}{ll}
\|u(t)\|_{L^{2}}=\|u(0)\|_{L^{2}}, & \text { (conservation of charge) } \\
E(u(t))=E(u(0)), & \text { (conservation of energy) }
\end{array}
$$

and

$$
\frac{d}{d t}\left(\|(x+2 i t \nabla) u(t)\|_{L^{2}}^{2}+\frac{8 \lambda t^{2}}{\alpha+2}\|u(t)\|_{L^{\alpha+2}}^{\alpha+2}\right)=4 \lambda \frac{4-N \alpha}{\alpha+2} t\|u(t)\|_{L^{x+2}}^{\alpha+2}
$$

where $E$ is defined by

$$
E(u(t))=\frac{1}{2} \int_{\mathbf{R}^{N}}|\nabla u(t, x)|^{2} d x+\frac{\lambda}{\alpha+2} \int_{\mathbf{R}^{N}}|u(t, x)|^{\alpha+2} d x .
$$

Conservation of charge and energy hold for all $H^{1}\left(\mathbf{R}^{N}\right)$ solutions, while (1.6), the "pseudo-conformal conservation law" is valid only for solutions in $X$. Note that this law is a true conservation law only if $\alpha=4 / N$. 
If $\lambda \geqq 0$, then all solutions of (1.2) are global and bounded in $H^{1}\left(\mathbf{R}^{N}\right)$. As well, if $\lambda<0$ but $\alpha<4 / N$, all solutions are global and bounded in $H^{1}\left(\mathbf{R}^{N}\right)$. If $\lambda<0$ and $\alpha \geqq 4 / N$, then all solutions with small initial data (in $H^{1}\left(\mathbf{R}^{N}\right)$ ) are global and bounded in $H^{1}\left(\mathbf{R}^{N}\right)$, but some solutions blow up in finite time $[10,15,24,4]$.

Concerning the scattering theory, several results are available. If $\alpha>4 / N$ and $\lambda>0$, then a scattering theory can be constructed in $H^{1}\left(\mathbf{R}^{N}\right)$, provided $N \geqq 3$. If $\lambda<0$, a similar theory can be constructed, but limited to small solutions $[12,13]$. If $\alpha>\alpha_{0}$, where

$$
\alpha_{0}=\frac{2-N+\sqrt{N^{2}+12 N+4}}{2 N},
$$

and $\lambda>0$, then the scattering theory can be constructed in $X[26,17]$. The same holds for small solutions if $\lambda<0[22,23]$. Note that $2 / N<\alpha_{0}<4 / N$. On the other hand, if $\alpha>2 / N$ and $\lambda>0$, then every solution with initial value in $X$ has a scattering state in $L^{2}\left(\mathbf{R}^{N}\right)$, that is $S(-t) u(t)$ has a strong limit in $L^{2}\left(\mathbf{R}^{N}\right)$, as $t \rightarrow \infty$ [27]. Finally, if $\alpha \leqq 2 / N$, then no scattering theory can be constructed since for any nontrivial solution $u$ of (1.1), $S(-t) u(t)$ does not have any strong limit in $L^{2}\left(\mathbf{R}^{N}\right)$ as $t \rightarrow \pm \infty$ $[14,21,22,1,27]$. However, there still remains a gap between $2 / N$ and $\alpha_{0}$ for the scattering theory in $X$ and between $2 / N$ and $4 / N$ for the scattering theory in $H^{1}\left(\mathbf{R}^{N}\right)$.

The main result of this paper is the existence of a scattering theory in $X$, i.e. asymptotic completeness, for (1.1) in the case $\lambda>0$ and $\alpha=\alpha_{0}$ (Theorems 4.9 and 4.10). Also, we extend the lower limit of $\alpha$ to $\frac{4}{N+2}$ for which a low energy scattering theory in $X$ exists (Theorem 4.2 and Corollary 4.3); and we prove that for these new values of $\alpha$ the wave operators are defined on all of $X$ (Proposition 4.6). Our proofs make use of the pseudo-conformal transformation for (1.1), which has previously been used by Yajima [29], Tsutsumi and Yajima [27] and Tsutsumi [26] to study the scattering properties of Schrödinger equations.

In connection with the decay properties of solutions to (1.1), we make the following definition.

Definition 1.1. Suppose $0<\alpha<\frac{4}{N-2}(0<\alpha<\infty$, if $N=1,2)$. A positively global solution $u$ of (1.1) has rapid decay (or is rapidly decaying) if

$$
\int_{0}^{\infty}\|u(t)\|_{L^{x+2}\left(\mathbf{R}^{N}\right)}^{a} d t<\infty
$$

where

$$
a=\frac{2 \alpha(\alpha+2)}{4-\alpha(N-2)} .
$$

In principle, the decay of $u(t)$ could be measured by $\|u(t)\|_{L^{r}\left(\mathbf{R}^{N}\right)}$ for any $r$ with $2<r<\frac{2 N}{N-2}$. We choose $r=\alpha+2$ since the energy of the solution explicitly involves the $L^{\alpha+2}\left(\mathbf{R}^{N}\right)$ norm, as well as the fact that the nonlinear term in (1.1) is a mapping from $L^{r}\left(\mathbf{R}^{N}\right)$ into its dual precisely when $r=\alpha+2$. The value of $a$ is 
determined by (1.1) in the following manner. If $u$ is a solution of (1.1), then for all $\gamma>0, u_{\gamma}$ is likewise a solution of $(1.1)$, where $u_{\gamma}(t, x)=\gamma^{2 / \alpha} u\left(\gamma^{2} t, \gamma x\right)$. We would like the parameter $a$ to be chosen so that the space-time integral (1.8) is invariant under the transformation $u \rightarrow u_{\gamma}$. A straightforward calculation shows that the correct value of $a$ is given by (1.9).

One of our principal results (Proposition 2.4) is that if $\alpha_{0}<\alpha<\frac{4}{N-2}$ and if $\varphi \in X$ is such that $\int_{0}^{\infty}\|S(t) \varphi\|_{L^{+2}}^{a} d t$ is sufficiently small, then the corresponding solution of (1.1) is positively global and has rapid decay. We deduce from this that if $b>0$ is sufficiently large, then the solution of (1.1) with initial value $\varphi(x) e^{i\left(b|x|^{2} / 4\right)}$ is positively global and has rapid decay (Corollary 2.5). In particular, if $\lambda<0$ and $\frac{4}{N} \leqq \alpha<\frac{4}{N-2}$, finite time blow up of solutions to (1.1) can not be deduced solely from properties of the modulus of the initial value.

Much of the recent work on (1.1) makes use of space-time estimates for the linear homogeneous and inhomogeneous Schrödinger equations. These estimates go back to Strichartz [25] and were first used systematically to study the nonlinear Schrödinger and Klein-Gordon equations by Ginibre and Velo [11-13]. Since then, these inequalities have been extended and generalized, for example in $[28,5,6,8,9,19]$. In this paper we use the following results of this type $[11,5,4]$.

Definition 1.2. An admissible pair in $\mathbf{R}^{N}$ is a pair $(q, r)$ of real numbers such that

(i) $2 \leqq r<\frac{2 N}{N-2}(2 \leqq r<\infty$, if $N=2 ; 2 \leqq r \leqq \infty$, if $N=1)$;

(ii) $\frac{2}{q}=N\left(\frac{1}{2}-\frac{1}{r}\right)$.

In particular, the pair $(\infty, 2)$ is always admissible. Note that, if $(q, r)$ is an admissible pair, then $q \in(2, \infty](q \in[4, \infty]$, if $N=1)$.

Proposition 1.3. The Schrödinger group $S(\cdot)$ satisfies the following properties.

(i) If $\varphi \in L^{2}\left(\mathbf{R}^{N}\right)$, then $S(\cdot) \varphi \in C\left(\mathbf{R}, L^{2}\left(\mathbf{R}^{N}\right)\right) \cap L^{q}\left(\mathbf{R} ; L^{r}\left(\mathbf{R}^{N}\right)\right)$ for every admissible pair $(q, r)$. Furthermore, there exists a constant $C$ depending only on $q$ such that

$$
\|S(\cdot) \varphi\|_{L^{q}\left(\mathbf{R}, L^{r}\right)} \leqq C\|\varphi\|_{L^{2}},
$$

for every $\varphi \in L^{2}\left(\mathbf{R}^{N}\right)$.

(ii) Given $0<T<\infty$ and $f \in L^{1}\left(0, T ; H^{-1}\left(\mathbf{R}^{N}\right)\right)$, let $\mathscr{F}_{f} \in C\left([0, T], H^{-1}\left(\mathbf{R}^{N}\right)\right)$ be defined by

$$
\mathscr{F}_{f}(t)=\int_{0}^{t} S(t-s) f(s) d s, \text { for all } t \in[0, T]
$$

If $(q, r)$ is an admissible pair and $f \in L^{q^{\prime}}\left(0, T ; L^{r^{\prime}}\left(\mathbf{R}^{N}\right)\right)$, then

$$
\mathscr{F}_{f} \in C\left([0, T], L^{2}\left(\mathbf{R}^{N}\right)\right) \cap L^{\gamma}\left(0, T ; L^{\rho}\left(\mathbf{R}^{N}\right)\right)
$$

for every admissible pair $(\gamma, \rho)$. In addition, there exists a constant $C$, depending only 
on $\gamma$ and $q$, such that

$$
\left\|\mathscr{F}_{f}\right\|_{L^{\gamma}\left(0, T ; L^{\rho}\right)} \leqq C\|f\|_{L^{q^{\prime}\left(0, T ; L^{r^{\prime}}\right)}},
$$

for every $f \in L^{q^{\prime}}\left(0, T ; L^{r^{\prime}}\left(\mathbf{R}^{N}\right)\right)$, where' denotes the conjugate exponent.

Definition 1.4. Suppose $0<\alpha<\frac{4}{N-2}(0<\alpha<\infty$, if $N=1,2)$. A positively global solution $u$ of (1.1) has linear decay if $u \in L^{q}\left(0, \infty ; L^{r}\left(\mathbf{R}^{N}\right)\right)$, where $(q, r)$ is the admissible pair such that $r=\alpha+2$. Note that $q=\frac{4(\alpha+2)}{N \alpha}$.

Note that linear decay and rapid decay coincide if and only if $\alpha=4 / N$. Moreover, for this value of $\alpha$, Eq. (1.1) has some special invariance properties not shared by the other powers. In particular, if $\alpha=4 / N$ and $u$ is a solution of (1.1), then $v$, defined by

$$
v(t, x)=(1+b t)^{-N / 2} u\left(\frac{t}{1+b t}, \frac{x}{1+b t}\right) e^{i\left(b|x|^{2} / 4(1+b t)\right)},
$$

is likewise a solution of (1.1). It follows that global and nonglobal solutions can be transformed into each other. In [7] we used this transformation to study decay and scattering properties, as well as finite time blow up, of solutions to (1.1) with $\alpha=4 / N$. Even though for other values of $\alpha,(1.1)$ is no longer invariant under the transformation (1.13), this transformation still provides a tool for studying global solutions of (1.1). As mentioned above, this idea has been effectively exploited in the works of Yajima [29], Tsutsumi and Yajima [27] and Tsutsumi [26].

If $u$ is a solution of (1.1), then $v$ defined by (1.13) satisfies a nonautonomous version of (1.1), namely

$$
i v_{t}+\Delta v=\lambda(1+b t)^{(N \alpha-4) / 2}|v|^{\alpha} v .
$$

Taking, for example, $b=-1$, one sees that $u$ defined on $[0, \infty)$ corresponds to $v$ defined on $[0,1)$. The study of the asymptotic behavior of $u$ as $t \rightarrow \infty$ is thereby reduced to studying the Cauchy problem for (1.14) at the (perhaps singular) point $t=1$. The local study of (1.14) is analogous to the local study of (1.1). The basic idea is to use the linear estimates in Proposition 1.3 to effect a contraction mapping argument for the corresponding integral equation. For Eq. (1.1) this theory has been developed in the papers $[11,18,6]$. For $(1.14)$, this theory has been developed by Tsutsumi and Yajima [27] and Tsutsumi [26], and has been constructed for $\alpha_{0}<\alpha<\frac{4}{N-2}$, where $\alpha_{0}$ is as in (1.7). In the present work, using a function space motivated by [6], we are able to bring down the local solvability from $\alpha_{0}$ to $\frac{4}{N+2}$.

See Theorem 3.4 for the precise statement, as well as Remark 3.5 (ii) for an explanation of the choice of function space used in the proof of local existence.

More interesting than the local study of (1.14) is the global study of (1.14), in other words the existence of solutions to (1.14), with $b=-1$, on the closed interval $[0,1]$. Up until now, it is known that if $\lambda \geqq 0$ and $\alpha_{0}<\alpha<\frac{4}{N-2}$, then all local solutions are global. By combining lower bounds for nonglobal solutions obtained 
from the local existence theorem for (1.14) with energy estimates for (1.14), we are able to prove that all solutions are global if $\lambda>0$ and $\alpha=\alpha_{0}$ (Proposition 3.12). This result is equivalent, via transformation (1.13), to asymptotic completeness.

We remark that any fact about solutions to (1.1) proved via the pseudo-conformal transformation can be proved directly. Such proofs have been given by Ginibre and Velo [10] and by Hayashi and Tsutsumi [17] for the previously known scattering results; and in principle the new results presented here could be proved similarly.

The rest of this paper is organized as follows. In Sect. 2 we study rapidly decaying solutions of (1.1). In Sect. 3, we study the Cauchy problem for the nonautonomous Equation (1.14) with $b<0$, which we can take for convenience to be -1 . In Sect. 4, we use the results of Sects. 2 and 3 to study the scattering theory for (1.1). In Sects. 3 and 4, we develop the scattering theory in $X$ in its entirety, rather than proving only our new results. Indeed, our treatment gives the previously known results with no extra work, and provides a unified exposition of the theory. In Sect. 5, we make some additional remarks, including a heuristic interpretation of finite time blow up of solutions to (1.1).

Throughout this paper we use the following conventions. All function spaces are spaces of complex valued functions. The number $\alpha$ always satisfies

$$
0<\alpha<\frac{4}{N-2} .
$$

It is understood that the second inequality means $\alpha<\infty$ if $N=1,2$. Finally, $\alpha_{0}$ always denotes the value given in (1.7).

\section{Rapidly Decaying Solutions}

We begin this section with a space-time inequality for $S(t)$ that involves nonadmissible pairs.

Lemma 2.1. Let $(q, r)$ be an admissible pair with $r>2$. Fix $a>\frac{q}{2}$ and define $\tilde{a}$ by

$$
\frac{1}{\tilde{a}}+\frac{1}{a}=\frac{2}{q} .
$$

If $T>0$ and $f \in L^{\tilde{L}^{\prime}}\left(0, T ; L^{r^{\prime}}\left(\mathbf{R}^{N}\right)\right)$, then $\mathscr{F}_{f} \in L^{a}\left(0, T ; L^{r}\left(\mathbf{R}^{N}\right)\right)$, where $\mathscr{F}_{f}$ is given by (1.11). Furthermore, there exists a constant $C$, depending only on $N$, $r$ and a such that

$$
\left\|\mathscr{F}_{f}\right\|_{L^{a}\left(0, T ; L^{r}\right)} \leqq C\|f\|_{L^{a^{\prime}}\left(0, T ; L^{r^{\prime}}\right)},
$$

for every $f \in L^{\tilde{a}^{\prime}}\left(0, T ; L^{r^{\prime}}\left(\mathbf{R}^{N}\right)\right)$.

Proof. By density, we only need to prove estimate (2.2) for $f \in C\left([0, T], \mathscr{S}\left(\mathbf{R}^{N}\right)\right)$. It is well known and follows easily from (1.3) by interpolation that

$$
\|S(t)\|_{\mathscr{L}\left(L^{\left.r^{\prime}, L^{r}\right)} \leqq\right.} \leqq\left. 4 \pi t\right|^{-N((1 / 2)-(1 / r))}
$$

whenever $2 \leqq r \leqq \infty$. Therefore,

$$
\left\|\mathscr{F}_{f}(t)\right\|_{L^{r}} \leqq \int_{0}^{t}(4 \pi(t-s))^{-2 / q}\|f(s)\|_{L^{r}} d s
$$


and so (2.2) is an immediate consequence of the Riesz potential inequalities ([20], Theorem 1, p. 119).

Lemma 2.2. Let $r=\alpha+2$, let $(q, r)$ be the corresponding admissible pair, and let a be given by (1.9). Then $a>q / 2$ if and only if $\alpha>\alpha_{0}$, with $\alpha_{0}$ defined by (1.7). For such values of $\alpha$ and $a$, and for $0<T \leqq \infty$, we have the following estimates for $\mathscr{F}$ defined by (1.11).

(i) If $u \in L^{a}\left(0, T ; L^{r}\left(\mathbf{R}^{N}\right)\right)$, then $\mathscr{F}_{|u|^{\mid}{ }_{u}} \in L^{a}\left(0, T ; L^{r}\left(\mathbf{R}^{N}\right)\right)$. Furthermore, there exists $C$ depending only on $N$ and $\alpha$ such that

$$
\left\|\mathscr{F}_{\left.||^{\mid}\right|_{u}}\right\|_{L^{a}\left(0, T ; L^{r}\right)} \leqq C\|u\|_{L^{a}\left(0, T ; L^{r}\right)}^{\alpha+1},
$$

for every $u \in L^{a}\left(0, T ; L^{r}\left(\mathbf{R}^{N}\right)\right)$.

(ii) If $u \in L^{a}\left(0, T ; L^{r}\left(\mathbf{R}^{N}\right)\right) \cap L^{q}\left(0, T ; W^{1, r}\left(\mathbf{R}^{N}\right)\right)$ and if $(\gamma, \rho)$ is any admissible pair, then

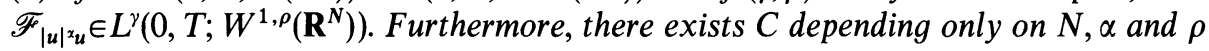
such that

$$
\left\|\mathscr{F}_{|u|^{x_{u}}}\right\|_{L^{\gamma\left(0, T ; W^{1, \rho)}\right.}} \leqq C\|u\|_{L^{a}\left(0, T ; L^{r}\right)}^{\alpha}\|u\|_{L^{q}\left(0, T ; W^{1, r}\right)},
$$

for every $u \in L^{a}\left(0, T ; L^{r}\left(\mathbf{R}^{N}\right)\right) \cap L^{q}\left(0, T ; W^{1, r}\left(\mathbf{R}^{N}\right)\right)$.

Proof. The first part of the lemma is a simple calculation, which we omit. For assertions (i) and (ii) consider $\tilde{a}$ defined by (2.1). Since $(\alpha+1) r^{\prime}=r,(\alpha+1) \tilde{a}^{\prime}=a$ and

$$
\frac{1}{q^{\prime}}=\frac{1}{q}+\frac{\alpha}{a}
$$

we see that

$$
\left\||u|^{\alpha} u\right\|_{L^{a^{\prime}\left(0, T ; L^{r^{\prime}}\right)}}=\|u\|_{L^{a}\left(0, T ; L^{r}\right)}^{\alpha+1}
$$

and (applying Hölder's inequality twice) that

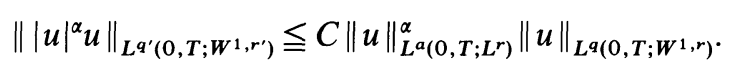

The results now follow from (2.2) and (1.12) respectively.

Proposition 2.3. Assume that $\alpha_{0}<\alpha<\frac{4}{N-2}$, where $\alpha_{0}$ is defined by (1.7). If $u \in$ $C\left([0, \infty), H^{1}\left(\mathbf{R}^{N}\right)\right)$ is a rapidly decaying solution of $(1.1)$, then $u \in L^{\gamma}\left(0, \infty ; W^{1, \rho}\left(\mathbf{R}^{N}\right)\right)$ for every admissible pair $(\gamma, \rho)$. In particular, $u$ has linear decay.

Proof. It is known $[18,6]$ that $u \in L^{\nu}\left(0, T ; W^{1, \rho}\left(\mathbf{R}^{N}\right)\right)$, for every $T<\infty$ and every admissible pair $(\gamma, \rho)$. For a fixed $T \geqq 0$, we set $v(t)=u(t+T)$; and so

$$
v(t)=S(t) u(T)-i \lambda \mathscr{F}_{|v|^{\alpha} v} .
$$

As before, set $r=\alpha+2$, and let $q$ be such that $(q, r)$ is an admissible pair. Let $(\gamma, \rho)$ be any admissible pair, and let $a$ be defined by (1.9). It follows from (1.10) and (2.4) that

$$
\|v\|_{L^{\gamma\left(0, \tau ; W^{1, \rho}\right)}} \leqq C_{1}\|u(T)\|_{H^{1}}+C_{2}\|u\|_{L^{a}\left(T, T+\tau ; L^{r}\right)}^{\alpha}\|v\|_{L^{q}\left(0, \tau ; W^{1, r}, r\right)},
$$

for every $\tau>0$. Choosing $T$ large enough so that $C_{2}\|u\|_{L^{a}\left(T, \infty ; L^{r}\right)}^{\alpha} \leqq 1 / 2$ and letting $(\gamma, \rho)=(q, r)$, we see that

$$
\|v\|_{L^{q}\left(0, \tau ; W^{1, r}\right)} \leqq 2 C_{1}\|u(T)\|_{H^{1}}
$$


for every $\tau>0$. Hence $u \in L^{q}\left(0, \infty ; W^{1, r}\left(\mathbf{R}^{N}\right)\right)$. That $u \in L^{\gamma}\left(0, \infty ; W^{1, \rho}\left(\mathbf{R}^{N}\right)\right)$ for any admissible pair $(\gamma, \rho)$ now follows from $(2.5)$.

Proposition 2.4. Assume $\alpha_{0}<\alpha<\frac{4}{N-2}$, where $\alpha_{0}$ is defined by (1.7), and let a be given by (1.9). There exists $\varepsilon>0$ such that if $\varphi \in H^{1}\left(\mathbf{R}^{N}\right)$ and $\|S(\cdot) \varphi\|_{L^{a}\left(0, \infty ; L^{\alpha+2}\right)} \leqq \varepsilon$, then the maximal solution $u$ of (1.1) with initial value $\varphi$ is positively global and has rapid decay.

Proof. Let $\varepsilon>0$ and set $r=\alpha+2$; let $\varphi \in H^{1}\left(\mathbf{R}^{N}\right)$ be such that $\|S(\cdot) \varphi\|_{L^{a}\left(0, \infty ; L^{r}\right)} \leqq \varepsilon$; and let $u$ be the maximal solution of $(1.1)$ with initial value $\varphi$, with $\left[0, T^{*}\right), 0<T^{*} \leqq \infty$, the maximal existence interval of $u$. Consider $q$ such that $(q, r)$ is an admissible pair. It follows from (2.3), (1.10) and (2.4) that there exists $K$ independent of $\varphi$ such that

and

$$
\|u\|_{L^{a}\left(0, T ; L^{r}\right)} \leqq \varepsilon+K\|u\|_{L^{a}\left(0, T ; L^{r}\right)}^{\alpha+1}
$$

$$
\|u\|_{L^{q}\left(0, T ; W^{1, r}\right)} \leqq K\|\varphi\|_{H^{1}}+K\|u\|_{L^{a}\left(0, T ; L^{r}\right)}^{\alpha}\|u\|_{L^{q}\left(0, T ; W^{1, r}\right)}
$$

for every $T<T^{*}$. Assume that $\varepsilon$ satisfies

$$
2^{\alpha+1} K \varepsilon^{\alpha}<1
$$

Since $\|u\|_{L^{a}\left(0, T ; L^{r}\right)}$ depends continuously on $T$, it follows from (2.6) and (2.8) that

Applying (2.7) and (2.9), we get

$$
\|u\|_{L^{a}\left(0, T^{*} ; L^{r}\right)} \leqq 2 \varepsilon
$$

$$
\|u\|_{L^{q}\left(0, T^{*} ; W^{1, r}\right)} \leqq 2 K\|\varphi\|_{H^{1}} .
$$

Applying now (1.10) and (2.4) with $(\gamma, \rho)=(\infty, 2)$, and using (2.9) and (2.10), we see that

$$
\|u\|_{L^{\infty}\left(0, T^{*} ; H^{1}\right)}<\infty .
$$

Therefore [18,5], $T^{*}=\infty$, and the result follows from (2.9) and Proposition 2.3.

Corollary 2.5. Assume that $\alpha_{0}<\alpha<\frac{4}{N-2}$, where $\alpha_{0}$ is defined by (1.7). Suppose $\varphi \in X$.

(i) There exists $b_{0}<\infty$ such that for every $b \geqq b_{0}$, the maximal solution of (1.1) with initial value $\varphi_{b}(x)=e^{i\left(b|x|^{2} / 4\right)} \varphi(x)$ is positively global and has rapid decay.

(ii) There exists $s_{0}<\infty$ such that for every $s \geqq s_{0}$, the maximal solution of (1.1) with initial value $\psi_{s}=S(s) \varphi$ is positively global and has rapid decay.

Proof. Since $\varphi \in X$, we have $\varphi_{b} \in X$, for every $b \in \mathbf{R}$. Let $(q, r)$ be the admissible pair such that $r=\alpha+2$ and let $a$ be defined by (1.9). Using the explicit kernel (1.3), one verifies that (see for example [7], formulas (3.2) and (3.3))

$$
\left\|S(\cdot) \varphi_{b}\right\|_{L^{a}\left(0, \infty ; L^{r}\right)}^{a}=\int_{0}^{1 / b}(1-b \tau)^{2(a-q) / q}\|S(\tau) \varphi\|_{L^{r}}^{a} d \tau
$$

Since $\|S(\tau) \varphi\|_{L^{r}} \leqq C\|S(\tau) \varphi\|_{H^{1}} \leqq C\|\varphi\|_{H^{1}}$ and $\frac{2(a-q)}{q}>-1$, it follows that

$$
\lim _{b \uparrow \infty}\left\|S(\cdot) \varphi_{b}\right\|_{L^{a}\left(0, \infty ; L^{r}\right)}=0 .
$$


By Proposition 2.4, this proves the first assertion. To prove the second assertion, it suffices to note that, since $\varphi \in X,\|S(\cdot) \varphi\|_{L^{a}\left(0, \infty ; L^{r}\right)}$ is finite.

Remark 2.6. Suppose that $\frac{4}{N} \leqq \alpha<\frac{4}{N-2}$ and that $\lambda<0$. If $\varphi \in X$ is such that $E(\varphi)<0$, then it is well known $[15,24,4]$ that the maximal solution $u$ of $(1.1)$ with initial value $\varphi$ blows up in finite time, for both $t>0$ and $t<0$. Corollary 2.5 implies that if $b$ is large enough, then the maximal solution $u_{b}$ of $(1.1)$ with initial value $\varphi_{b}(x)=$ $\varphi(x) e^{i\left(b|x|^{2} / 4\right)}$ is positively global and has rapid decay. Of course, $E\left(\varphi_{b}\right) \geqq 0$ for such $b$ 's, and one may wonder if $u_{b}$ still blows up at a finite negative time. The answer is yes, as the following argument shows. Changing $\varphi_{b}$ to $\overline{\varphi_{b}}$ (which changes $u_{b}(t)$ to $\left.\overline{u_{b}(-t)}\right)$, it suffices to show that if $E(\varphi)<0$, then for all $b>0$ the solution $u$ of $(1.1)$ with initial value $\varphi(x) e^{-i\left(b|x|^{2} / 4\right)}$ blows up at a positive finite time. Let $T^{*}$ be the maximal existence time of $u$, and let $f(t)=\||\cdot| u(t, \cdot)\|_{L^{2}}^{2}$. It is well known $[10,4]$ that $f \in C^{2}\left(\left[0, T^{*}\right)\right)$

$$
f^{\prime}(t)=4 \operatorname{Im} \int_{\mathbf{R}^{N}} x \bar{u} \nabla u d x
$$

and

$$
f^{\prime \prime}(t)=16 E(u(0))+\lambda \frac{4(N \alpha-4)}{\alpha+2} \int_{\mathbf{R}^{N}}|u|^{\alpha+2}
$$

Therefore,

$$
f(t)=f(0)+t f^{\prime}(0)+8 E(u(0)) t^{2}+\lambda \frac{4(N \alpha-4)}{\alpha+2} \int_{0}^{t} \int_{0}^{s} \int_{\mathbf{R}^{N}}|u|^{\alpha+2} d x d \sigma d s,
$$

for all $0 \leqq t<T^{*}$. It follows that

$$
f(t) \leqq f(0)+t f^{\prime}(0)+8 E(u(0)) t^{2}
$$

for all $0 \leqq t<T^{*}$. Setting $P(t)=f(0)+t f^{\prime}(0)+8 E(u(0)) t^{2}$ for all $t \geqq 0$, a straightforward calculation (see [7], formulas (4.3) through (4.7)) shows that

$$
P(t)=\|x \varphi\|_{L^{2}}^{2}+4 t\left(F(\varphi)-\frac{b}{2}\|x \varphi\|_{L^{2}}^{2}\right)+8 t^{2}\left(E(\varphi)+\frac{b^{2}}{8}\|x \varphi\|_{L^{2}}^{2}-\frac{b}{2} F(\varphi)\right),
$$

with

$$
F(\varphi)=\operatorname{Im} \int_{\mathbf{R}^{N}} x \bar{\varphi} \nabla \varphi d x
$$

In particular,

$$
P(1 / b)=\frac{8}{b^{2}} E(\varphi)<0 ;
$$

and it follows easily from $(2.12)$ that $T^{*}<1 / b$. Hence the result.

Proposition 2.7. Assume that $4 / N<\alpha<\frac{4}{N-2}$, and set $r=\alpha+2$. If $u \in C([0, \infty)$, $\left.H^{1}\left(\mathbf{R}^{N}\right)\right)$ is a global solution of (1.1) such that $\|u(t)\|_{L^{r}} \underset{t \rightarrow \infty}{\longrightarrow} 0$, then $u$ has linear and rapid decay. More generally, $u \in L^{\gamma}\left(0, \infty ; W^{1, \rho}\left(\mathbf{R}^{N}\right)\right)$ for every admissible pair $(\gamma, \rho)$. 
Proof. Let $q$ be such that $(q, r)$ is an admissible pair and let $T \geqq 0$. We have

$$
u(T+t)=S(t) u(T)-i \lambda \int_{0}^{t} S(t-s)|u|^{\alpha} u(T+s) d s .
$$

It follows from (1.10) and (1.12), applied with $(\gamma, \rho)=(q, r)$, that

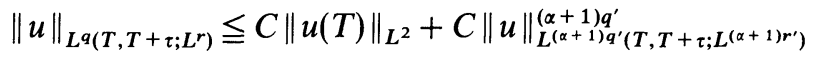

$$
\begin{aligned}
& \leqq C\|u(0)\|_{L^{2}}+C\left(\int_{T}^{T+\tau}\|u(s)\|_{L^{r}}^{(\alpha+1) q^{\prime}} d s\right)^{1 / q^{\prime}},
\end{aligned}
$$

for every $\tau>0$. Since $\alpha>4 / N$ we see that $(\alpha+1) q^{\prime}>q$. Therefore, if $\delta=(\alpha+1) q^{\prime}-q$, then

$$
\|u\|_{L^{q}\left(T, T+\tau ; L^{r}\right)} \leqq C\|u(0)\|_{L^{2}}+C \sup \left\{\|u(s)\|_{L^{r}} ; s \geqq T\right\}^{\delta / q^{\prime}}\|u\|_{L^{q}\left(T, T+\tau ; L^{r}\right)}^{q / q^{\prime}} .
$$

Thus, if $T$ is large enough so that

it follows easily that

$$
C \sup \left\{\|u(s)\|_{L^{r}} ; s \geqq T\right\}^{\delta / q^{\prime}}\left(C\|u(0)\|_{L^{2}}\right)^{q-2}<1,
$$

$$
\|u\|_{L^{q}\left(T, T+\tau ; L^{r}\right)} \leqq 2 C\|u(0)\|_{L^{2}},
$$

for every $\tau>0$. Therefore, $u \in L^{q}\left(0, \infty ; L^{r}\left(\mathbf{R}^{N}\right)\right)$, i.e. $u$ has linear decay. Since $\alpha>4 / N$ and $\|u(t)\|_{L^{r}} \rightarrow 0$ as $t \rightarrow \infty$, it follows that $u$ has rapid decay. The last assertion follows from Proposition 2.3.

\section{The Nonautonomous Equation}

We now apply the "pseudo-conformal transformation" (1.13) with $b<0$, and we suppose for convenience that $b=-1$. Moreover, throughout this section we systematically consider the variables $(s, y) \in \mathbf{R} \times \mathbf{R}^{N}$ defined by

$$
s=\frac{t}{1-t}, \quad y=\frac{x}{1-t},
$$

or equivalently,

$$
t=\frac{s}{1+s}, \quad x=\frac{y}{1+s} .
$$

Given $0 \leqq a<b \leqq \infty$ and $u$ defined on $(a, b) \times \mathbf{R}^{N}$, we set

$$
v(t, x)=(1-t)^{-N / 2} u\left(\frac{t}{1-t}, \frac{x}{1-t}\right) e^{-i\left(|x|^{2} / 4(1-t)\right)},
$$

for $x \in \mathbf{R}^{N}$ and $\frac{a}{1+a}<t<\frac{b}{1+b}$. In particular, if $u$ is defined on $(0, \infty)$, then $v$ is defined on $(0,1)$. Transformation (3.1) reads as well, using the variables $(s, y)$,

$$
v(t, x)=(1+s)^{N / 2} u(s, y) e^{-i\left(|y|^{2} / 4(1+s)\right)} .
$$

One verifies easily that, given $0 \leqq a<b<\infty, u \in C([a, b], X) \cap C^{1}\left([a, b], H^{-1}\left(\mathbf{R}^{N}\right)\right)$ 
if and only if $v \in C\left(\left[\frac{a}{1+a}, \frac{b}{1+b}\right], X\right) \cap C^{1}\left(\left[\frac{a}{1+a}, \frac{b}{1+b}\right], H^{-1}\left(\mathbf{R}^{N}\right)\right)$. Furthermore, a straightforward calculation shows that $u$ solves $(1.1)$ on $(a, b)$ if, and only if $v$ solves equation

$$
i v_{t}+\Delta v=\lambda(1-t)^{(N \alpha-4) / 2}|v|^{\alpha} v,
$$

on the interval $\left(\frac{a}{1+a}, \frac{b}{1+b}\right)$. Note that the term $(1-t)^{(N \alpha-4) / 2}$ is regular, except possibly at $t=1$, where it is singular for $\alpha<4 / N$. One verifies easily the following identities:

$$
\begin{aligned}
& \|v(t)\|_{L^{\beta+2}}^{\beta+2}=(1+s)^{N \beta / 2}\|u(s)\|_{L^{\beta+2}}^{\beta+2}, \quad \beta \geqq 0, \\
& \|\nabla v(t)\|_{L^{2}}^{2}=\frac{1}{4}\|(y+2 i(1+s) \nabla) u(s)\|_{L^{2}}^{2}, \\
& \|\nabla u(s)\|_{L^{2}}^{2}=\frac{1}{4}\|(x+2 i(1-t) \nabla) v(t)\|_{L^{2}}^{2} .
\end{aligned}
$$

The point of view we take in this section is to consider Eq. (3.3) in its own right, and study the associated Cauchy problem for any starting time in $[0,1]$. Near the end of this section, we interpret some of the results for solutions of (3.3) in terms of solutions of (1.1). In Sect. 4, we will use the information gathered in this section about solutions to (3.3) to deduce information about the scattering properties of solutions to (1.1). Our proof of local existence of solutions to the Cauchy problem for Eq. (3.3) is based on the following lemma.

Lemma 3.1. Assume $0<\alpha<\frac{4}{N-2}$. Let $\theta=\frac{4}{4-\alpha(N-2)}(\theta=1$, if $N=1 ; \theta>1$ and $(2-\alpha) \theta \leqq 1$, if $N=2)$, and consider a real valued function $h \in L_{\mathrm{loc}}^{\theta}(\mathbf{R})$. Then, for every $\psi \in H^{1}\left(\mathbf{R}^{\bar{N}}\right)$, there exist $T_{*}<0<T^{*}$ and a unique, maximal solution $v \in C\left(\left(T_{*}, T^{*}\right)\right.$, $\left.H^{1}\left(\mathbf{R}^{N}\right)\right) \cap W_{\text {loc }}^{1, \theta}\left(T_{*}, T^{*} ; H^{-1}\left(\mathbf{R}^{N}\right)\right)$ of equation

$$
v(t)=S(t) \psi-i \int_{0}^{t} S(t-s) h(s)|v|^{\alpha} v(s) d s
$$

The solution $v$ is maximal in the sense that if $T^{*}<\infty$ (respectively $T_{*}>-\infty$ ), then $\|u(t)\|_{H^{1}} \rightarrow \infty$, as $t \uparrow T^{*}$ (respectively $t \downarrow T_{*}$ ). In addition, the solution $v$ has the following properties.

(i) If $T^{*}<\infty$, then $\liminf _{t \uparrow T^{*}}\left\{\|v(t)\|_{H^{1}}^{\alpha}\|h\|_{L^{\theta}\left(t, T^{*}\right)}\right\}>0$.

(ii) If $T_{*}>-\infty$, then $\liminf _{t \uparrow T^{*}}\left\{\|v(t)\|_{H^{1}}^{\alpha}\|h\|_{L^{\theta}\left(T_{*}, t\right)}\right\}>0$.

(iii) $u \in L_{\text {loc }}^{q}\left(T_{*}, T^{*} ; W^{1, r}\left(\mathbf{R}^{N}\right)\right)$, for every admissible pair $(q, r)$.

(iv) There exists $\delta>0$, depending only on $N$, $\alpha$ and $\theta$ such that if

$$
\|\psi\|_{\boldsymbol{H}^{1}}^{\alpha \theta} \int_{-\tau}^{\tau}|h(s)|^{\theta} d s \leqq \delta
$$

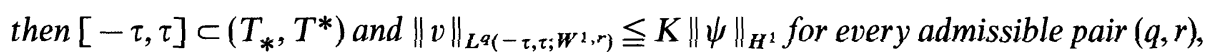
where $K$ depends only on $N, \alpha, \theta$ and $q$. In addition, if $\psi^{\prime}$ is another initial value satisfying the above condition and if $v^{\prime}$ is the corresponding solution of (3.7), then $\left\|v-v^{\prime}\right\|_{L^{\infty}\left(-\tau, \tau ; L^{2}\right)} \leqq K\left\|\psi-\psi^{\prime}\right\|_{L^{2}}$. 
(v) If $\psi \in X$, then $v \in C\left(\left(T_{*}, T^{*}\right), X\right)$. In addition,

$$
\|x v(t)\|_{L^{2}}^{2}=\|x \psi\|_{L^{2}}^{2}+4 \operatorname{Im} \int_{0}^{t} \int_{\mathbf{R}^{N}} \overline{v(s)}(x \cdot \nabla v(s)) d x d s,
$$

for all $t \in\left(T_{*}, T^{*}\right)$.

(vi) $\|v(t)\|_{L^{2}}=\|\psi\|_{L^{2}}$, for all $t \in\left(T_{*}, T^{*}\right)$.

Proof. For technical reasons, we suppose first that $N \geqq 3$. Afterwards, we will indicate the modifications needed to handle the cases $N=2$ and $N=1$. Let $2 *=\frac{2 N}{N-2}$, and
define $r$ by

$$
1-\frac{2}{r}=\frac{\alpha}{2 *}
$$

Since $(N-2) \alpha<4$, it follows that $2<r<2 *$. Therefore, there exists $q$ such that $(q, r)$ is an admissible pair. A simple calculation shows that

$$
\frac{1}{q^{\prime}}=\frac{1}{\theta}+\frac{1}{q}
$$

By Proposition 1.3, there exists $K$ such that

$$
\|S(\cdot) \psi\|_{L^{\infty}\left(\mathbf{R} ; H^{1}\right)}+\|S(\cdot) \psi\|_{L^{q}\left(\mathbf{R} ; W^{1, r}\right)} \leqq K\|\psi\|_{H^{1}},
$$

for every $\psi \in H^{1}\left(\mathbf{R}^{N}\right)$. Given $M>0$ and $T_{1} \leqq 0 \leqq T_{2}$ such that $T_{2}>T_{1}$, let

$$
\begin{array}{r}
E=\left\{v \in C\left(\left[T_{1}, T_{2}\right], H^{1}\left(\mathbf{R}^{N}\right)\right) \cap L^{q}\left(T_{1}, T_{2} ; W^{1, r}\left(\mathbf{R}^{N}\right)\right) ;\right. \\
\left.\|v\|_{L^{\infty}\left(T_{1}, T_{2} ; H^{1)}\right.}+\|v\|_{L^{q}\left(T_{1}, T_{2} ; W^{1, r}\right)} \leqq(K+1) M\right\} .
\end{array}
$$

Endowed with the metric $d(u, v)=\|v-u\|_{L^{q}\left(T_{1}, T_{2} ; L^{r}\right)},(E, d)$ is a complete metric space. Given $v \in E$, it follows from (3.8), (3.9), Sobolev's and Hölder's inequalities that $h|v|^{\alpha} v \in L^{q^{\prime}}\left(T_{1}, T_{2} ; W^{1, r^{\prime}}\left(\mathbf{R}^{N}\right)\right)$ and that

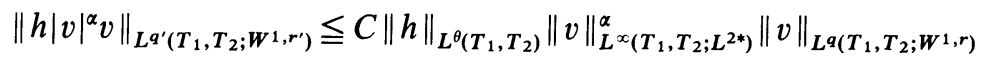

$$
\begin{aligned}
& \leqq C_{1}\|h\|_{L^{\theta}\left(T_{1}, T_{2}\right)}(K+1)^{\alpha+1} M^{\alpha+1} .
\end{aligned}
$$

Furthermore, given $u, v \in E$, one has as well

$$
\begin{aligned}
& \left\|h\left(|v|^{\alpha} v-|u|^{\alpha} u\right)\right\|_{L^{q^{\prime}\left(T_{1}, T_{2} ; L^{\prime}\right)}} \\
& \leqq C\|h\|_{L^{\theta}\left(T_{1}, T_{2}\right)}\left(\|v\|_{L^{\infty}\left(T_{1}, T_{2} ; H^{1}\right)}^{\alpha}+\|u\|_{L^{\infty}\left(T_{1}, T_{2} ; H^{1}\right)}^{\alpha}\right)\|v-u\|_{L^{q}\left(T_{1}, T_{2} ; L^{r}\right)}^{\alpha} ;
\end{aligned}
$$

and so,

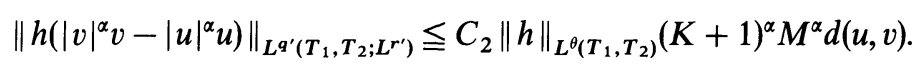

Given $v \in E$ and $\psi \in H^{1}\left(\mathbf{R}^{N}\right)$ such that $\|\psi\|_{H^{1}} \leqq M$, set

$$
\mathscr{G}_{v}(t)=S(t) \psi-i \int_{0}^{t} S(t-s) h(s)|v|^{\alpha} v(s) d s
$$

for $t \in\left(T_{1}, T_{2}\right)$. It follows from Proposition 1.3 and formula (3.10) that

$$
\mathscr{G}_{v} \in C\left(\left[T_{1}, T_{2}\right], H^{1}\left(\mathbf{R}^{N}\right)\right) \cap L^{q}\left(T_{1}, T_{2} ; W^{1, r}\left(\mathbf{R}^{N}\right)\right),
$$


and that

$$
\left\|\mathscr{G}_{v}\right\|_{L^{\infty}\left(T_{1}, T_{2} ; H^{1}\right)}+\left\|\mathscr{G}_{v}\right\|_{L^{q}\left(T_{1}, T_{2} ; W^{1, r}\right)} \leqq K M+C_{3}(K+1)^{\alpha+1} M^{\alpha+1}\|h\|_{L^{\theta}\left(T_{1}, T_{2}\right)} .
$$

Therefore, if $T_{2}-T_{1}$ is small enough so that

$$
C_{3}(K+1)^{\alpha+1} M^{\alpha}\|h\|_{L^{\theta}\left(T_{1}, T_{2}\right)} \leqq 1,
$$

then $\mathscr{G}_{v} \in E$. Furthermore, Proposition 1.3 and formula (3.12) imply that

$$
d\left(\mathscr{G}_{v}, \mathscr{G}_{u}\right) \leqq C_{4}(K+1)^{\alpha} M^{\alpha}\|h\|_{L^{\theta}\left(T_{1}, T_{2}\right)} d(u, v) .
$$

Consequently, if $T_{2}-T_{1}$ is small enough so that

$$
K_{1} M^{\alpha}\|h\|_{L^{\theta}\left(T_{1}, T_{2}\right)} \leqq 1 / 2,
$$

where $K_{1}=(K+1)^{\alpha+1} \max \left\{C_{3}, C_{4}\right\}$, then $\mathscr{G}$ is Lipschitz continuous $E \rightarrow E$ with Lipschitz constant $1 / 2$. Therefore, $\mathscr{G}$ has a unique fixed point $v \in E$, which solves Eq. (3.7). In addition, the first part of property (iv) follows from (3.13), (3.10) and Proposition 1.3, and the second part from (3.11) and Proposition 1.3. Uniqueness in the class $C\left(\left[T_{1}, T_{2}\right] ; H^{1}\left(\mathbf{R}^{N}\right)\right)$ follows from (3.11) and Proposition 1.3. (Note that uniqueness is a local property and needs only to be established for $T_{2}-T_{1}$ small enough.) Now, by uniqueness, $v$ can be extended to a maximal interval $\left(T_{*}, T^{*}\right)$, and property (iii) follows from property (iv). Suppose that $T^{*}<\infty$. Applying the above local existence result to $v(t), t<T^{*}$ with $T_{1}=0$, we see from (3.13) that if

$$
K_{1}\|v(t)\|_{H^{1}}^{\alpha}\|h\|_{L^{\theta}\left(t, T^{*}\right)} \leqq 1 / 2,
$$

then $v$ can be continued up to and beyond $T^{*}$, which is a contradiction. Therefore, we have

$$
K_{1}\|v(t)\|_{H^{1}}^{\alpha}\|h\|_{L^{\theta}\left(t, T^{*}\right)}>1 / 2,
$$

which proves property (i). Property (ii) is proved by the same argument. Finally, since $v$ solves the equation

$$
i v_{t}+\Delta v=h|v|^{\alpha} v
$$

in $L_{\mathrm{loc}}^{\theta}\left(T_{*}, T^{*} ; H^{-1}\left(\mathbf{R}^{N}\right)\right.$ and $h$ is real valued, property $(\mathrm{v})$ is proved by standard arguments. For example, multiply the above equation by $|x|^{2} e^{-\varepsilon|x|^{2}} \bar{v}$, take the imaginary part and integrate over $\mathbf{R}^{N}$, then let $\varepsilon \downarrow 0$ (see Proposition 6.4.2, p. 107 in [4] for a similar argument). Property (vi) follows by taking the $H^{1}-H^{-1}$ duality product of the equation with $v$.

If $N=2$, the proof is the same as in the case $N \geqq 3$, except that we set $r=2 \theta$ and use the embedding $H^{1}\left(\mathbf{R}^{2}\right) \subset L^{p}\left(\mathbf{R}^{2}\right)$ with $p=\frac{\alpha \theta}{\theta-1}$.

If $N=1$, the argument is slightly simpler. We let

$$
E=\left\{v \in C\left(\left[T_{1}, T_{2}\right], H^{1}(\mathbf{R})\right) ;\|v\|_{L^{\infty}\left(T_{1}, T_{2} ; H^{1}\right)} \leqq 2 M\right\},
$$

equipped with the metric $d(u, v)=\|v-u\|_{L^{\infty}\left(T_{1}, T_{2} ; H^{1}\right)}$, and use the embedding $H^{1}(\mathbf{R}) \subseteq L^{\infty}(\mathbf{R})$.

Corollary 3.2. Under the assumptions of Lemma 3.1, suppose there exists $\theta_{1}>\theta$ such that $h \in L_{\text {loc }}^{\theta_{1}}(\mathbf{R})$. It follows that for every $M>0$, there exists $\tau>0$ such that if $\psi \in H^{1}\left(\mathbf{R}^{N}\right)$ 
verifies $\|\psi\|_{H^{1}}<M$, then $[-\tau, \tau] \subset\left(T_{*}, T^{*}\right)$, and such that $v$ has the following continuity properties.

(i) If $\|\psi\|_{H^{1}}<M, \psi_{n} \underset{n \rightarrow \infty}{\longrightarrow} \psi$ in $H^{1}\left(\mathbf{R}^{N}\right)$, and if $v_{n}$ denotes the solution of (3.7) with initial value $\psi_{n}$, then $v_{n} \rightarrow$ in $C\left([-\tau, \tau], H^{1}\left(\mathbf{R}^{N}\right)\right)$.

(ii) If $\|\psi\|_{H^{1}}<M, \psi_{n} \rightarrow \infty$ in $X$, and if $v_{n}$ denotes the solution of (3.7) with initial value $\psi_{n}$, then $v_{n} \rightarrow v$ in $C([-\tau, \tau], X)$.

Proof. We base our proof on arguments of Kato [18] used in the autonomous case. We only prove the result in the case $N \geqq 3$ (see the proof of Lemma 3.1 for the necessary modifications in the cases $N=1,2$ ). Given $M>0$, we choose $\tau$ so that the inequality in property (iv) of Lemma 3.1 is met whenever $\|\psi\|_{H^{1}}<M$. In particular, if $\|\psi\|_{H^{1}}<M$, then $[-\tau, \tau] \subset\left(T_{*}, T^{*}\right)$. Next, observe that $\frac{1}{\theta}>\frac{4-\alpha N}{4}$; and so we may assume without loss of generality that $\frac{1}{\theta_{1}}>\frac{4-\alpha N}{4}$. Therefore, if we define $\sigma$ by

$$
\frac{1}{\sigma}=\frac{2}{N}\left(1-\frac{1}{\theta_{1}}\right)
$$

then $2<\alpha \sigma<\frac{2 N}{N-2}$. Let now $\rho$ be defined by

$$
1-\frac{2}{\rho}=\frac{1}{\sigma} \text {. }
$$

Since $\frac{1}{\sigma}<\frac{2}{N}$, it follows that $2<\rho<\frac{2 N}{N-2}$. Finally, let $\gamma$ be such that $(\gamma, \rho)$ is an admissible pair. It follows easily from Hölder's inequality that for every $-\infty<a<$ $b<\infty$,

$$
\|h w z\|_{L^{\gamma^{\prime}\left(a, b ; L^{\rho^{\prime}}\right)}} \leqq\left(\int_{a}^{b} h(s)^{\theta_{1}}\|w(s)\|_{L^{\sigma^{\prime}}}^{\theta_{1}}\right)^{1 / \theta_{1}}\|z\|_{L^{\prime}\left(a, b ; L^{\prime}\right)} .
$$

Consider now $\psi$ such that $\|\psi\|_{H^{1}} \leqq M$, and let $\psi_{n}$ be as in (i). Let $v, v_{n}$ be the corresponding solutions of (3.7). It follows from Proposition 1.3 that there exists $C$, depending only on $\gamma$, such that

$$
\begin{aligned}
& \left\|v-v_{n}\right\|_{L^{\gamma\left(-\tau, \tau ; W^{1, \rho)}\right.}}+\left\|v-v_{n}\right\|_{L^{\infty}\left(-\tau, \tau ; H^{1}\right)} \\
& \quad \leqq C\left\|\psi-\psi_{n}\right\|_{H^{1}}+\left\|h\left(|v|^{\alpha} v-\left|v_{n}\right|^{\alpha} v_{n}\right)\right\|_{L^{\gamma^{\prime}}\left(-\tau, \tau ; W^{1,}, \rho^{\prime}\right.} .
\end{aligned}
$$

On the other hand, a straightforward calculation shows that

$$
\left|\nabla\left(|v|^{\alpha} v-\left|v_{n}\right|^{\alpha} v_{n}\right)\right| \leqq C\left|v_{n}\right|^{\alpha}\left|\nabla v-\nabla v_{n}\right|+\phi\left(v, v_{n}\right)|\nabla v|,
$$

where $C$ depends on $\alpha$, and the function $\phi(x, y)$ is bounded by $C\left(|x|^{\alpha}+|y|^{\alpha}\right)$ and verifies $\phi(x, y) \underset{y \rightarrow x}{\longrightarrow} 0$. Therefore, applying (3.14), (3.15) and (3.16), we get

$$
\begin{aligned}
& \left\|v-v_{n}\right\|_{L^{\gamma\left(-\tau, \tau ; W^{1, \rho)}\right.}}+\left\|v-v_{n}\right\|_{L^{\infty}\left(-\tau, \tau ; H^{1}\right)} \\
& \leqq \\
& \quad+C \psi-\psi_{n}\left\|_{H^{1}}+\right\| h\left\|_{L^{\theta_{1}(-\tau, \tau)}}\right\| v_{n}\left\|_{L^{\infty}\left(-\tau, \tau ; L^{x \sigma}\right)}\right\| v-v_{n} \|_{L^{\gamma\left(-\tau, \tau ; W^{1, \rho)}\right.}} \\
& \quad+C\left(\int_{a}^{b} h(s)^{\theta_{1}}\left\|\phi\left(v, v_{n}\right)\right\|_{L^{\sigma}}^{\theta_{1}}\right)^{1 / \theta_{1}}\|v\|_{L^{\gamma\left(-\tau, \tau ; W^{1, \rho)}\right.}} .
\end{aligned}
$$


Note that by property (iv) of Lemma 3.1, $v_{n}$ is bounded in $H^{1}\left(\mathbf{R}^{N}\right)$, hence in $L^{\alpha \sigma}\left(\mathbf{R}^{N}\right)$, with the bound, for $t \in[-\tau, \tau]$, depending only on $\left\|\psi_{n}\right\|_{H^{1}}$, hence (for large values of $n$ ) only on $M$. As well, the bound on $\|v\|_{L^{\gamma\left(-\tau, \tau ; W^{1}, \rho\right)}}$ depends only on $M$. Therefore, it follows from (3.17) that

$$
\begin{aligned}
& \left\|v-v_{n}\right\|_{L^{\gamma}\left(-\tau, \tau ; W^{1, \rho)}\right.}+\left\|v-v_{n}\right\|_{L^{\infty}\left(-\tau, \tau ; H^{1}\right)} \\
& \quad \leqq C\left\|\psi-\psi_{n}\right\|_{H^{1}}+C\|h\|_{L^{\theta_{1}(-\tau, \tau)}}\left\|v-v_{n}\right\|_{L^{\gamma\left(-\tau, \tau ; W^{1, \rho}\right)}}+C\left(\int_{a}^{b} h(s)^{\theta_{1}}\left\|\phi\left(v, v_{n}\right)\right\|_{L^{\sigma}}^{\theta_{1}}\right)^{1 / \theta_{1}},
\end{aligned}
$$

where the constant $C$ depends only on $M$. Therefore, if we consider $\tau$ possibly smaller so that $C\|h\|_{L^{\theta_{1}(-\tau, \tau)}} \leqq 1 / 2$ (note that $\tau$ still depends on $M$ ), it follows that

$$
\begin{aligned}
& \left\|v-v_{n}\right\|_{L^{\gamma\left(-\tau, \tau ; W^{1, \rho}\right)}}+\left\|v-v_{n}\right\|_{L^{\infty}\left(-\tau, \tau ; H^{1}\right)} \\
& \quad \leqq C\left\|\psi-\psi_{n}\right\|_{H^{1}}+C\left(\int_{a}^{b} h(s)^{\theta_{1}}\left\|\phi\left(v, v_{n}\right)\right\|_{L^{\sigma}}^{\theta_{1}}\right)^{1 / \theta_{1}} .
\end{aligned}
$$

Therefore, property (i) follows, provided we show that

$$
\left(\int_{a}^{b} h(s)^{\theta_{1}}\left\|\phi\left(v, v_{n}\right)\right\|_{L^{\sigma}}^{\theta_{1}}\right) \underset{n \rightarrow \infty}{\longrightarrow} 0 .
$$

By the dominated convergence theorem, it suffices to verify that

$$
\left\|\phi\left(v, v_{n}\right)\right\|_{L^{\sigma}} \underset{n \rightarrow \infty}{\longrightarrow} 0
$$

for all $t \in[-\tau, \tau]$. To see this, we argue by contradiction. We assume that there exists $t$ and a subsequence, which we still denote by $v_{n}(t)$, such that $\left\|\phi\left(v(t), v_{n}(t)\right)\right\|_{L^{\sigma}} \geqq \mu>0$. Note that $v_{n}(t) \rightarrow v(t)$ in $L^{2}\left(\mathbf{R}^{N}\right)$ and $v_{n}(t)$ is bounded in $H^{1}\left(\mathbf{R}^{N}\right)$ by property (iv) of Lemma 3.1. Therefore, by Sobolev's and Hölder's inequalities, $v_{n}(t) \rightarrow v(t)$ in $L^{\alpha \sigma}\left(\mathbf{R}^{N}\right)$. It follows that there exists a subsequence, which we still denote by $v_{n}(t)$, and a function $f \in L^{\alpha \sigma}\left(\mathbf{R}^{N}\right)$ such that $v_{n}(t) \rightarrow v(t)$ almost everywhere in $\mathbf{R}^{N}$ and $\left|v_{n}(t)\right| \leqq f$ almost everywhere in $\mathbf{R}^{N}$. Applying the dominated convergence theorem, it follows that $\left\|\phi\left(v(t), v_{n}(t)\right)\right\|_{L^{\sigma}} \rightarrow 0$, which is a contradiction. Hence property (i).

Property (ii) follows from property (i) and Lemma 3.1 (v). Briefly, use Hölder's inequality on the formula in Lemma 3.1 (v) to obtain a uniform bound in $X$ on the solutions $v_{n}$. The integral term then converges along subsequences where $x v_{n}$ converges weakly in $L^{2}\left(\mathbf{R}^{N}\right)$ to $x v$, and the rest of the proof is standard.

Corollary 3.3. Under the assumptions of Corollary 3.2, the solution $v$ of (3.7) given by Lemma 2.1 depends continuously on $\psi$ in the following way.

(i) The mapping $\psi \mapsto T^{*}$ is lower semicontinuous $H^{1}\left(\mathbf{R}^{N}\right) \rightarrow(0, \infty]$.

(ii) The mapping $\psi \mapsto T_{*}$ is upper semicontinuous $H^{1}\left(\mathbf{R}^{N}\right) \rightarrow[-\infty, 0)$.

(iii) If $\psi_{n} \underset{n \rightarrow \infty}{\longrightarrow} \psi$ in $H^{1}\left(\mathbf{R}^{N}\right)$ and if $\left[T_{1}, T_{2}\right] \in\left(T_{*}, T^{*}\right)$, then $v_{n} \rightarrow$ vin $C\left(\left[T_{1}, T_{2}\right], H^{1}\left(\mathbf{R}^{N}\right)\right)$,

where $v_{n}$ denotes the solution of (3.7) with initial value $\psi_{n}$. If in addition $\psi_{n} \rightarrow \psi$ in $X$, then $v_{n} \rightarrow v$ in $C\left(\left[T_{1}, T_{2}\right], X\right)$.

Proof. Let $\psi \in H^{1}\left(\mathbf{R}^{N}\right)$, let $v$ be the maximal solution of (3.7) given by Lemma 2.1 and let $\left[T_{1}, T_{2}\right] \subset\left(T_{*}, T^{*}\right)$. Set $M=\frac{1}{2} \sup _{T_{1} \leqq t \leqq T_{2}}\|v(t)\|_{H^{1}}$ and consider $\tau>0$ given by Corollary 3.2. By applying Corollary $3.2 m$ times, where $(m-1) \tau<T_{2}-T_{1} \leqq m \tau$, 
we see that if $\|\psi-\psi\|_{H^{1}}$ is small enough, then the solution of (3.7) with initial value $\widetilde{\psi}$ exists on $\left[T_{1}, T_{2}\right]$. Hence properties (i) and (ii). Property (iii) follows easily from the same argument.

We are now in a position to state our local existence result for Eq. (3.3). In order to make the statement simpler, we introduce the function

$$
f(t)=\lambda|1-t|^{-((4-N \alpha) / 2)}, \text { for } t \in \mathbf{R},
$$

and the equation

$$
v(t)=S\left(t-t_{0}\right) \psi-i \int_{t_{0}}^{t} S(t-s) f(s)|v|^{\alpha} v(s) d s
$$

where $t_{0} \in \mathbf{R}$ and $\psi \in H^{1}\left(\mathbf{R}^{N}\right)$. Note that if $0 \leqq T_{1} \leqq t_{0} \leqq T_{2}<1$, then $v$ solves (3.18) on $\left[T_{1}, T_{2}\right]$ if and only if $v$ solves (3.3) on $\left[T_{1}, T_{2}\right]$ with initial value $v\left(t_{0}\right)=\psi$.

Theorem 3.4. Assume $\frac{4}{N+2}<\alpha<\frac{4}{N-2}(2<\alpha<\infty$, if $N=1)$. Then, for every $t_{0} \in \mathbf{R}$ and $\psi \in H^{1}\left(\mathbf{R}^{N}\right)$, there exist $T_{*}<t_{0}<T^{*}$ and a unique, maximal solution $v \in C\left(\left(T_{*}, T^{*}\right)\right.$, $H^{1}\left(\mathbf{R}^{N}\right)$ ) of Eq. (3.18). The solution $v$ is maximal in the sense that if $T^{*}<\infty$ (respectively $\left.T_{*}>-\infty\right)$, then $\|u(t)\|_{H^{1}} \rightarrow \infty$, as $t \uparrow T^{*}$ (respectively $t \downarrow T_{*}$ ). In addition, the solution $v$ has the following properties.

(i) If $T^{*}=1$, then $\liminf _{t \uparrow 1}\left\{(1-t)^{\delta}\|v(t)\|_{H^{1}}\right\}>0$ with $\delta=\frac{N+2}{4}-\frac{1}{\alpha}$ if $N \geqq 3$, $\delta$ any number larger than $1-\frac{1}{\alpha}$ if $N=2$, and $\delta=\frac{1}{2}-\frac{1}{\alpha}$ if $N=1$.

(ii) If $\psi \in X$, then $v \in C\left(\left(T_{*}, T^{*}\right), X\right)$.

(iii) $\|v(t)\|_{L^{2}}=\left\|v\left(t_{0}\right)\right\|_{L^{2}}$, for all $t \in\left(T_{*}, T^{*}\right)$.

(iv) The solution $v$ depends continuously on $\psi$ in the following way. The mapping $\psi \mapsto T^{*}$ is lower semicontinuous $H^{1}\left(\mathbf{R}^{N}\right) \rightarrow(0, \infty]$, and the mapping $\psi \mapsto T_{*}$ is upper semicontinuous $H^{1}\left(\mathbf{R}^{N}\right) \rightarrow[-\infty, 0)$. In addition, if $\psi_{n} \underset{n \rightarrow \infty}{\longrightarrow} \psi$ in $H^{1}\left(\mathbf{R}^{N}\right)$ and if $\left[T_{1}, T_{2}\right] \in$ $\left(T_{*}, T^{*}\right)$, then $v_{n} \rightarrow v$ in $C\left(\left[T_{1}, T_{2}\right], H^{1}\left(\mathbf{R}^{N}\right)\right)$, where $v_{n}$ denotes the solution of $(3.18)$ with initial value $\psi_{n}$. If in addition $\psi_{n} \rightarrow \psi$ in $X$, then $v_{n} \rightarrow v$ in $C\left(\left[T_{1}, T_{2}\right], X\right)$.

Proof. The result is a straightforward consequence of Lemma 3.1 and Corollary 3.3 with $h(t)=f\left(t+t_{0}\right)$.

Remark 3.5. (i) Note that even when $\alpha \leqq \frac{4}{N+2}(\alpha \leqq 2$, if $N=1)$ the nonautonomous term in (3.3) is singular only at $t=1$. Therefore, by applying Lemma 3.1, one can still solve Eq. (3.3) away from $t=1$. More precisely, since in this case all solutions of (1.1) with initial values in $X$ are global, it follows that for every $\psi \in X$ and for every $t_{0} \in[0,1)$, there exists a unique solution $v \in C([0,1), X)$ of $(3.3)$ such that $v\left(t_{0}\right)=\psi$.

(ii) It is the choice of $r$ given by (3.8) in the proof of Lemma 3.1 which enables us to prove local existence starting from $t=1$ for (3.18) if $\frac{4}{N+2}<\alpha<\frac{4}{N-2}$. If instead one uses $r=\alpha+2$, the range of $\alpha$ is restricted to $\alpha_{0}<\alpha<\frac{4}{N-2}$. 
(iii) The lower bound $\alpha>2$ in dimension $N=1$ corresponds to $\alpha>\frac{2}{N}$.

Proposition 3.6. Assume $\frac{4}{N+2}<\alpha<\frac{4}{N-2}(2<\alpha<\infty$, if $N=1)$. Let $t_{0}, t_{1} \in[0,1]$ and let $\mathscr{E}$ be the set of $\psi \in X$ such that the solution $v$ of (3.18) with initial value $v\left(t_{0}\right)=\psi$ exists on the interval $J_{\left(t_{0}, t_{1}\right)}=\left[\min \left\{t_{0}, t_{1}\right\}, \max \left\{t_{0}, t_{1}\right\}\right]$. Then, $\mathscr{E}$ is an open subset of $X$ containing 0. Moreover, the mapping $U: \psi \mapsto v$ is continuous $\mathscr{E} \rightarrow C\left(J_{\left(t_{0}, t_{1}\right)}, X\right)$.

Proof. The result follows from property (iv) of Theorem 3.4.

Corollary 3.7. Assume $\frac{4}{N+2}<\alpha<\frac{4}{N-2}(2<\alpha<\infty$, if $N=1)$. Let $\mathscr{E}$ be the set of $\psi \in X$ such that the solution $v$ of (3.18) with initial value $v(0)=\psi$ exists at $t=1$, i.e. $T^{*}>1 . \mathscr{E}$ then has the following properties.

(i) $\mathscr{E}$ is an open subset of $X$ containing 0 .

(ii) The mapping $U: \psi \mapsto v(1)$ is continuous $\mathscr{E} \rightarrow X$.

(iii) The set $\mathscr{F}=U(\mathscr{E})$ is an open subset of $X$ containing 0 and $U: \mathscr{E} \rightarrow \mathscr{F}$ is one to one.

(iv) $U^{-1}$ is continuous $\mathscr{F} \rightarrow \mathscr{E}$.

Proof. Properties (i) and (ii) follow by applying Proposition 3.6 with $t_{0}=0$ and $t_{1}=1$. The fact that $U$ is one to one follows from the uniqueness property of Theorem 3.4. The other properties follow by applying Proposition 3.6 with $t_{0}=1$ and $t_{1}=0$.

Proposition 3.8. Assume that $0<\alpha<\frac{4}{N-2}$. Let $0 \leqq t_{0}<t_{1}<1$, and suppose that $v \in C\left(\left[t_{0}, t_{1}\right], X\right)$ solves Eq. (3.3) on $\left[t_{0}, t_{1}\right]$. For $t \in\left[t_{0}, t_{1}\right]$, let

$$
\begin{gathered}
E_{1}(t)=\frac{1}{2}\|\nabla v(t)\|_{L^{2}}^{2}+(1-t)^{(N \alpha-4) / 2} \frac{\lambda}{\alpha+2}\|v(t)\|_{L^{\alpha+2}}^{\alpha+2}, \\
E_{2}(t)=(1-t)^{(4-N \alpha) / 2} E_{1}(t)=(1-t)^{(4-N \alpha) / 2} \frac{1}{2}\|\nabla v(t)\|_{L^{2}}^{2}+\frac{\lambda}{\alpha+2}\|v(t)\|_{L^{\alpha+2}}^{\alpha+2},
\end{gathered}
$$

and

$$
E_{3}(t)=\frac{1}{8}\|(x+2 i(1-t) \nabla) v(t)\|_{L^{2}}^{2}+(1-t)^{N \alpha / 2} \frac{\lambda}{\alpha+2}\|v(t)\|_{L^{x+2}}^{\alpha+2} .
$$

Then, $E_{1}, E_{2}, E_{3} \in C^{1}\left(\left[t_{0}, t_{1}\right]\right)$ and

$$
\begin{gathered}
\frac{d}{d t} E_{1}(t)=(1-t)^{(N \alpha-6) / 2} \frac{4-N \alpha}{2} \frac{\lambda}{\alpha+2}\|v(t)\|_{L^{\alpha+2}}^{\alpha+2}, \\
\frac{d}{d t} E_{2}(t)=(1-t)^{(2-N \alpha) / 2} \frac{N \alpha-4}{4}\|\nabla v(t)\|_{L^{2}}^{2},
\end{gathered}
$$

and

for all $t \in\left[t_{0}, t_{1}\right]$.

$$
\frac{d}{d t} E_{3}(t)=0
$$


Proof. Let $u$ be defined by (3.2). It follows from (3.4) and (3.5) that

$$
E_{1}(t)=\frac{1}{8}\|(y+2 i(1+s) \nabla) u(s)\|^{2}+(1+s)^{2} \frac{\lambda}{\alpha+2}\|u(s)\|_{L^{\alpha+2}}^{\alpha+2} .
$$

Formula (3.19) now follows from the pseudo-conformal conservation law (1.6) applied to $u(\cdot-1)$. Formula (3.20) follows immediately from (3.19); and (3.21) follows from (1.5), (3.4) and (3.6).

Remark 3.9. Consider $\psi \in X, t_{0} \in[0,1)$, and let $v \in C\left(\left(T_{*}, T^{*}\right), X\right)$ be the maximal solution of (3.18). The following properties of $T^{*}$ and $T_{*}$ are analogous to global existence properties of the corresponding solution $u$ of (1.1) given by (3.1).

(i) If $\alpha<4 / N$, then $T_{*}<0$ and $T^{*} \geqq 1$. This follows rather easily from formulas (3.19) and (3.20), Gagliardo-Nirenberg's inequality and conservation of charge or, alternatively, from the fact that all solutions $u$ of (1.1) are global in this case.

(ii) If $\lambda \geqq 0$ and $\alpha \geqq 4 / N$, then $T_{*}<0$ and $T^{*}>1$. The two inequalities follow from formulas (3.20) and (3.19), respectively.

(iii) Note that if $\lambda<0$, then for any value of $\alpha, 0<\alpha<\frac{4}{N-2}$ there exist initial values $\psi$ for which $T^{*}=1$. Indeed, if $u(t, x)=e^{i \omega t} \varphi(x)$ is a standing wave of $(1.1)$ (see $[3,2]$ ), then one verifies easily that the corresponding solution $v$ of (3.3) blows up at $t=1$.

We will now investigate sufficient conditions for a solution $v$ of (3.3) to exist at $t=1$, i.e. $T^{*}>1$.

Proposition 3.10. Assume $\alpha_{0}<\alpha<\frac{4}{N-2}$, where $\alpha_{0}$ is defined by (1.7). Let $v \in$ $C([0,1), X)$ be a solution of $(3.18)$, and let $T^{*} \geqq 1$ be the maximal existence time of $v$.

(i) If $(1-t)^{(N \alpha-4) / 2 \alpha} v \in L^{a}\left(0,1 ; L^{\alpha+2}\left(\mathbf{R}^{N}\right)\right)$, with a given by $(1.9)$, then $T^{*}>1$.

(ii) If $\|v(t)\|_{L^{\alpha+2}}$ is bounded as $t \uparrow 1$, then $T^{*}>1$.

(iii) If $\lambda \geqq 0$, then $T^{*}>1$.

Proof. Suppose $(1-t)^{(N \alpha-4) / 2 \alpha} v \in L^{a}\left(0,1 ; L^{\alpha+2}\left(\mathbf{R}^{N}\right)\right)$. Set $r=\alpha+2$, and let $(q, r)$ be the corresponding admissible pair. Given $0 \leqq t_{0} \leqq t<1$, it follows from Eq. (3.18) and Proposition 1.3 that

$\|v\|_{L^{\infty}\left(t_{0}, t ; H^{1}\right)}+\|v\|_{L^{q}\left(t_{0}, t ; W^{1, r}\right)} \leqq C\left\|v\left(t_{0}\right)\right\|_{H^{1}}+C\left\|(1-s)^{(N \alpha-4) / 2}|v|^{\alpha} v\right\|_{L^{q^{\prime}}\left(t_{0}, t ; W^{1, r^{\prime}}\right)}$.

Applying Hölder's inequality, we obtain

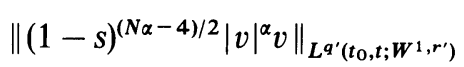

$$
\begin{aligned}
& \leqq C\left\|(1-s)^{(N \alpha-4) / 2}|v|^{\alpha}\right\|_{L^{q /(q-2)}\left(t_{0}, t ; L^{(\alpha+2) / \alpha}\right)}\|v\|_{L^{q\left(t_{0}, t ; W^{1, r}\right)}} .
\end{aligned}
$$

Since $\frac{\alpha q}{q-2}=a$, it follows that

$$
\left\|(1-s)^{(N \alpha-4) / 2}|v|^{\alpha} v^{\left(q^{\prime}\left(t_{0}, t ; W^{1, r^{\prime}}\right)\right.} \leqq C\right\|(1-s)^{(N \alpha-4) / 2 \alpha} v\left\|_{L^{a}\left(t_{0}, t ; L^{\alpha+2}\right)}^{\alpha}\right\| v \|_{L^{q}\left(t_{0}, t ; W^{1, r}\right)}
$$

and so,

$$
\begin{aligned}
& \|v\|_{L^{\infty}\left(t_{0}, t ; H^{1}\right)}+\|v\|_{L^{q}\left(t_{0}, t ; W^{1, r}\right)} \\
& \quad \leqq C\left\|v\left(t_{0}\right)\right\|_{H^{1}}+C\left\|(1-S)^{(N \alpha-4) / 2 \alpha} v\right\|_{L^{a}\left(t_{0}, t ; L^{x+2}\right)}^{\alpha}\|v\|_{L^{q}\left(t_{0}, t ; W^{1, r}\right)} .
\end{aligned}
$$


Choosing $t_{0}$ close enough to 1 so that $C\left\|(1-s)^{(N \alpha-4) / 2 \alpha} v\right\|_{L^{a}\left(t_{0}, 1 ; L^{\alpha+2}\right)}^{\alpha} \leqq 1 / 2$, we get

$$
\|v\|_{L^{\infty}\left(t_{0}, t ; H^{1}\right)}+\|v\|_{L^{q}\left(t_{0}, t ; W^{1, r}\right)} \leqq 2 C\left\|v\left(t_{0}\right)\right\|_{H^{1}} .
$$

It follows that $v$ remains bounded in $H^{1}\left(\mathbf{R}^{N}\right)$ as $t \uparrow 1$, and property (i) follows from Theorem 3.4. Since $\alpha>\alpha_{0}$, we have $\frac{N \alpha-4}{2 \alpha} a>-1$; and so, property (ii) follows from property (i). Finally, suppose that $\lambda \geqq 0$. If $\alpha<4 / N$, it follows from (3.20) that $v$ is bounded in $L^{\alpha+2}\left(\mathbf{R}^{N}\right)$. Therefore, $T^{*}>1$ by property (ii). If $\alpha \geqq 4 / N$, it follows from Remark 3.9 (ii) that $T^{*}>1$. Hence (iii). This completes the proof.

Remark 3.11. Note that in the proof of property (i) of Proposition 3.10 above, we did not make use of the assumption $\alpha>\alpha_{0}$. The same argument shows indeed that, if $\frac{4}{N+2}<\alpha \leqq \alpha_{0}\left(2<\alpha \leqq \alpha_{0}\right.$, if $\left.N=1\right)$ and if

$$
\int_{0}^{1}(1-t)^{a((N \alpha-4) / 2 \alpha)}\|v(t)\|_{L^{\alpha+2}}^{a} d t<\infty,
$$

then $T^{*}>1$. However, in this case, we have $\|v(t)\|_{L^{x+2}} \rightarrow\|v(1)\|_{L^{x+2}}$. If $v \neq \equiv$, it follows that the above integral is infinite, since $a \frac{N \alpha-4}{2 \alpha} \leqq-1$. This is absurd; and so, if $\frac{4}{N+2}<\alpha \leqq \alpha_{0}$, then

$$
\int_{0}^{1}(1-t)^{a((N \alpha-4) / 2 \alpha)}\|v(t)\|_{L^{\alpha+2}}^{a} d t=\infty,
$$

for every nontrivial solution $v \in C\left([0,1), H^{1}\left(\mathbf{R}^{N}\right)\right)$ of (3.18).

Proposition 3.12. Assume that $N \geqq 3$ and that $\alpha=\alpha_{0}$, where $\alpha_{0}$ is defined by (1.7). Let $v \in C([0,1), X)$ be a solution of $(3.18)$, and let $T^{*} \geqq 1$ be the maximal existence time of $v$.

(i) If $\limsup _{t \uparrow 1} \lambda|\log (1-t)|^{-1}\|v(t)\|_{L^{\alpha+2}}^{\alpha+2} \geqq 0$, then $T^{*}>1$.

(ii) If $\lambda \geqq 0$, then $T^{*}>1$.

Proof. We first prove assertion (i). Suppose to the contrary that $T^{*}=1$. It follows from Theorem 3.4 that there exists $\mu>0$ such that

$$
\|\nabla v(t)\|_{L^{2}}^{2} \geqq \mu(1-t)^{2 / \alpha-(N+2) / 2} .
$$

Therefore, applying (3.20) we get

$$
\frac{d}{d t} E_{2}(t) \leqq-\mu \frac{4-N \alpha}{4}(1-t)^{-1}
$$

and so,

$$
E_{2}(t) \leqq E(0)-\mu \frac{4-N \alpha}{4}|\log (1-t)|
$$

This contradicts the hypothesis of (i), proving (i). Property (ii) follows immediately. 
Remark 3.13. The above argument does not apply to the cases $N=1$ and $N=2$, since the lower estimate at blow up given by Theorem 3.4 is not good enough.

Finally, we will need the following results in the next section.

Proposition 3.14. Assume that $0<\alpha<\frac{4}{N-2}$, let $u \in C([0, \infty), X)$ be a solution of Eq. (1.1), and let $v \in C([0,1), X)$ be the corresponding solution of (3.3) defined by (3.2). Then, $S(-s) u(s)$ has a strong limit in $X$ (respectively, in $L^{2}\left(\mathbf{R}^{N}\right)$ ) as $s \rightarrow \infty$, if, and only if, $v(t)$ has a strong limit in $X$ (respectively, in $\left.L^{2}\left(\mathbf{R}^{N}\right)\right)$ as $t \uparrow 1$, in which case $\lim _{s \rightarrow \infty} S(-s) u(s)=e^{i\left(|x|^{2}\right) / 4} S(-1) v(1)$.

Proof. It follows from [7], formula (3.8) that

$$
S(-s) u(s)=e^{i\left(|x|^{2}\right) / 4} S\left(-\frac{s}{1+s}\right) v\left(\frac{s}{1+s}\right),
$$

from which the result follows.

Proposition 3.15. Assume that $\frac{4}{N+2}<\alpha<\frac{4}{N-2}$, let $u \in C([0, \infty), X)$ be a solution of Eq. (1.1), and let $v \in C([0,1), X)$ be the corresponding solution of (3.3) defined by (3.2). Let $T^{*} \geqq 1$ be the existence time of the maximal extension of $v$ as a solution of (3.18).

(i) If $T^{*}>1$, then $s^{(\mathrm{N \alpha}) / 2(\alpha+2)}\|u(s)\|_{L^{\alpha+2}}$ is bounded as $s \rightarrow \infty$. In particular, $u$ has linear decay, and if $\alpha_{0}<\alpha<\frac{4}{N-2}$, then $u$ has rapid decay.

(ii) If $\frac{4}{N+2}<\alpha \leqq \alpha_{0}\left(2<\alpha \leqq \alpha_{0}\right.$, if $\left.N=1\right)$, $u$ can not have rapid decay, except if $u \equiv 0$.

(iii) If $\alpha_{0}<\alpha<\frac{4}{N-2}$, and if $u$ has rapid decay, then $T^{*}>1$.

(iv) If $\alpha_{0}<\alpha<\frac{4}{N-2}$, and $\lambda \geqq 0$, then $u$ has both rapid and linear decay.

Proof. If $T^{*}>1$, then $\|v(t)\|_{L^{x+2}}$ is bounded as $t \uparrow 1$; and so, property (i) follows from identity (3.4). Property (ii) is a straightforward consequence of Remark 3.11 and identity (3.4). Suppose now that $\alpha>\alpha_{0}$ and that $u$ has rapid decay. Applying (3.4), we get $(1-t)^{(N \alpha-4) / 2 \alpha} v \in L^{a}\left(0,1 ; L^{\alpha+2}\left(\mathbf{R}^{N}\right)\right)$, with $a$ given by (1.9); and so, $T^{*}>1$ by Proposition 3.10. Hence (iii). Finally, (iv) follows from Proposition 3.10 and property (i).

Remark 3.16. In fact, it is already known (see for example [4]) that if $\lambda>0$ and $\alpha<\frac{4}{N-2}$, then $s^{(N \alpha) / 2(\alpha+2)}\|u(s)\|_{L^{\alpha+2}}$ is bounded as $s \rightarrow \infty$ for all solutions $u \in C([0, \infty), X)$ of $(1.1)$. 


\section{Applications to Scattering Theory}

Let $0<\alpha<\frac{4}{N-2}$. Given $\varphi \in X$, let $u_{\varphi}$ denote the maximal solution of (1.1) with initial value $u(0)=\varphi$. Let us set

and

$$
\begin{aligned}
& \mathscr{G}_{+}=\left\{\varphi \in X ; u_{\varphi} \text { exists on }[0, \infty)\right\}, \\
& \mathscr{G}_{-}=\left\{\varphi \in X ; u_{\varphi} \text { exists on }(-\infty, 0]\right\},
\end{aligned}
$$

For $\varphi \in \mathscr{R}_{+}$, let

$$
\mathscr{R}_{ \pm}=\left\{\varphi \in \mathscr{G}_{ \pm} ; S(-s) u_{\varphi}(s) \text { has a limit in } X \text { as } s \rightarrow \pm \infty\right\}
$$

where the limit holds in $X$. Finally, let us set

$$
\mathscr{S}_{ \pm}=U_{ \pm}\left(\mathscr{R}_{ \pm}\right) \text {. }
$$

We begin with the following elementary result.

Lemma 4.1. The sets $\mathscr{G}_{ \pm}, \mathscr{R}_{ \pm}$, and $\mathscr{S}_{ \pm}$have the following properties.

(i) $\mathscr{G}_{-}=\overline{\mathscr{G}_{+}}=\left\{\varphi ; \bar{\varphi} \in \mathscr{G}_{+}\right\}$;

(ii) $\mathscr{R}_{-}=\overline{\mathscr{R}_{+}}=\left\{\varphi ; \bar{\varphi} \in \mathscr{R}_{+}\right\}$;

(iii) $\mathscr{S}_{-}=\overline{\mathscr{S}_{+}}=\left\{\varphi ; \bar{\varphi} \in \mathscr{S}_{+}\right\}$;

(iv) $U_{-}(\varphi)=\overline{U_{+}(\bar{\varphi})}$, for every $\varphi \in \mathscr{R}_{-}$.

Proof. Note that $u(t, x)$ solves $(1.1)$ on $[0, T)$ if, and only if $w(t, x)=\overline{u(-t, x)}$ solves (1.1) on $(-T, 0]$. In other words, $u_{\varphi}(s)=\overline{u_{\bar{\varphi}}(-s)}$. Hence (i). Furthermore, since $S(-s) z=\overline{S(s) \bar{z}}$, we have

$$
S(-s) u_{\varphi}(s)=\overline{S(s) \overline{u_{\varphi}(s)}}=\overline{S(s) u_{\bar{\varphi}}(-s)}
$$

from which properties (ii), (iii) and (iv) follow immediately.

The following theorem concerning the wave and scattering operators is the main result of this section. Theorem 4.2. If $\frac{4}{N+2}<\alpha<\frac{4}{N-2}(2<\alpha<\infty$, if $N=1)$, then the following properties
hold.

(i) The sets $\mathscr{R}_{ \pm}$and $\mathscr{S}_{ \pm}$are open subsets of $X$, containing 0 .

(ii) The operators $U_{+}: \mathscr{R}_{ \pm} \rightarrow \mathscr{S}_{ \pm}$are bicontinuous bijections for the $X$ norm.

(iii) The wave operators $\Omega_{ \pm}=U_{ \pm}^{-1}$ are bicontinuous bijections $\mathscr{S}_{ \pm} \rightarrow \mathscr{R}_{ \pm}$for the $X$ norm.

(iv) The sets $\mathscr{O}_{+}=U_{+}\left(\mathscr{R}_{+} \cap \mathscr{R}_{-}\right)$and $\mathscr{O}_{-}=U_{-}\left(\mathscr{R}_{+} \cap \mathscr{R}_{-}\right)=\left\{z \in X ; \bar{z} \in \mathcal{O}_{+}\right\}$are open subsets of $X$ containing 0 .

(v) The scattering operator $\mathbf{S}=U_{+} \Omega_{-}: \mathcal{O}_{-} \rightarrow \mathcal{O}_{+}$is a bicontinuous bijection for the $X$ norm.

(vi) $\mathbf{S}^{-1}(z)=\overline{\mathbf{S}(\bar{z})}$, for every $z \in \mathcal{O}_{-}$. 
Proof. Let us first prove properties (i) and (ii). In view of Lemma 4.1, it is sufficient to establish these properties for the sets and operators indexed by + . These two properties now follow from Corollary 3.7 and Proposition 3.14 by interpreting the results in terms of $u$ given by the transformation (3.2). Property (iii) is a consequence of (ii). The identity $\mathcal{O}_{-}=\left\{z \in X ; \bar{z} \in \mathcal{O}_{+}\right\}$follows from Lemma 4.1, and the open character of $\mathcal{O}_{ \pm}$follows easily from property (ii). Finally, (v) is an immediate consequence of (ii) and (iii); and (vi) follows from Lemma 4.1.

The following result is an immediate consequence of Theorem 4.2.

Corollary 4.3. If $\frac{4}{N+2}<\alpha<\frac{4}{N-2}(2<\alpha<\infty$, if $N=1)$, then the scattering operator is defined from an $X$-neighborhood of 0 onto an $X$-neighborhood of 0 . In particular, a low energy scattering theory exists in $X$.

Remark 4.4. If $\lambda<0$, then the lower bound on $\alpha$ given in Theorem 4.2 for the existence of scattering states is optimal. Indeed, if $\alpha<\frac{4}{N+2}$, there exist initial data $\varphi$ of arbitrary small $X$-norm such that the solution $u$ of (1.1) with initial value $\varphi$ does not possess a scattering state in $X$ (or even in $L^{2}\left(\mathbf{R}^{N}\right)$ ). To see this, let $\varphi \in X$ be a nontrivial solution of the equation $-\Delta \varphi+\varphi=-\lambda|\varphi|^{\alpha} \varphi[3,2]$. Given $a>0$, set $\varphi_{a}(x)=a^{2 / \alpha} \varphi(a x)$. It follows that $-\Delta \varphi_{a}+a^{2} \varphi_{a}+\lambda\left|\varphi_{a}\right|^{\alpha} \varphi_{a}=0$. Therefore, $u_{a}(t, x)=$ $e^{i a^{2} t} \varphi_{a}(x)$ solves (1.1), and $S(-t) u_{a}(t)=e^{i a^{2} t} S(-t) \varphi_{a}$ does not have any strong limit as $t \rightarrow \infty$, even in $L^{2}\left(\mathbf{R}^{N}\right)$. On the other hand, one verifies easily that if $\alpha<\frac{4}{N+2}$, then $\left\|u_{a}\right\|_{X} \underset{a \downarrow 0}{\longrightarrow} 0$. The same example shows that for $\alpha<4 / N$, there exist initial data of arbitrary small $H^{1}$-norm such that the corresponding solution of (1.1) does not possess any scattering state (see Strauss [23]).

Remark 4.5. The conclusion of Corollary 4.3 was essentially proved by Strauss $[22,23]$ for $\alpha>\alpha_{0}$. Since $\frac{4}{N+2}<\alpha_{0}$, our result improves the range of $\alpha$.

Proposition 4.6. Assume that $\frac{4}{N+2}<\alpha<\frac{4}{N-2}(2<\alpha<\infty$, if $N=1)$. If $\lambda<0$, assume further that $\alpha<4 / N$. Then, $\mathscr{S}_{ \pm}=X$. Therefore, the wave operators are defined on all of $X$.

Proof. By Lemma 4.1, we need only show that $\mathscr{S}_{+}=X$. By transformation (3.2) and Proposition 3.14, it suffices to show that for every $w \in X$, there exists a solution $v$ of (3.3) that exists on $[0,1)$ and verifies $v(t) \underset{t \uparrow 1}{\longrightarrow} w$ in $X$. Now, given $w \in X$, it follows from Theorem 3.4 that there exists a unique solution $v$ of (3.3) on some interval $(1-\tau, 1)$ such that $v(t) \underset{t \uparrow 1}{\longrightarrow} w$ in $X$. By Remark 3.9,v exists on $[0,1)$. This proves the result

Remark 4.7. Note that when $\lambda<0$, the upper bound $\alpha<4 / N$ in Proposition 4.6 for the existence of the wave operators is optimal. Indeed, by Remark 2.6 there exists $\phi \in X$ such that the maximal solution $w$ of (1.1) with initial value $\phi$ has rapid decay at $+\infty$ and blows up at the negative, finite time $-\tau$. Let $w^{+}=\lim _{t \rightarrow \infty} S(-t) w(t)$ (see 
Theorem $4 / 12$ below), and set $u^{+}=S(-\tau) w^{+}$and $u(t)=w(t-\tau)$. It follows that $u^{+}=\lim _{t \rightarrow \infty} S(-t) u(t)$. Since $u(0)$ is not defined, it follows that $\Omega_{+} u^{+}$is not defined; and so, $\mathscr{S}_{+} \neq X$.

Remark 4.8. Existence of the wave operators has already been proved by Tsutsumi [26] (see also [17]) for $\alpha>\alpha_{0}$ in the case $\lambda>0$. Since $\frac{4}{N+2}<\alpha_{0}$, Proposition 4.6
improves the lower bound on $\alpha$.

We now study asymptotic completeness, and we begin with the case $\lambda \geqq 0$.

Theorem 4.9. If $N \geqq 3, \lambda \geqq 0$, and $\alpha_{0} \leqq \alpha<\frac{4}{N-2}$, where $\alpha_{0}$ is defined by (1.7), then
$\mathscr{R}_{ \pm}=X$.

Proof. By Lemma 4.1, we need only show that $\mathscr{R}_{+}=X$. Let $\psi \in X$. If follows from Proposition 3.10 (if $\alpha>\alpha_{0}$ ) and Proposition 3.12 (if $\alpha=\alpha_{0}$ ) that the solution $v$ of (3.18) with initial value $v(0)=\psi$ exists beyond $t=1$, i.e. $T^{*}>1$. The result now follows from Proposition 3.14.

Theorem 4.10. If $N \geqq 3, \lambda \geqq 0$, and $\alpha_{0} \leqq \alpha<\frac{4}{N-2}$, where $\alpha_{0}$ is defined by (1.7), then $\mathcal{O}_{ \pm}=X$. In particular, the scattering operator is a bicontinuous bijection $X \rightarrow X$.

Proof. This follows from Theorem 4.2, Proposition 4.6 and Theorem 4.9.

Remark 4.11. (i) The previous two results are valid for $N=1,2$ if $\alpha_{0}<\alpha$.

(ii) The conclusion of Theorem 4.10 was previously proved by Tsutsumi [26] (see also [17]) for $\alpha>\alpha_{0}$. However, the case $\alpha=\alpha_{0}$ is new.

We now characterize the sets $\mathscr{R}_{ \pm}$in the case $\lambda<0$ in terms of rapidly decaying solutions.

Theorem 4.12. If $\lambda<0$ and $\alpha_{0}<\alpha<\frac{4}{N-2}$, where $\alpha_{0}$ is defined by (1.7), then $\mathscr{R}_{+}=$ $\left\{\varphi \in X ; u_{\varphi}\right.$ has rapid decay $\}$ and $\mathscr{R}_{-}=\left\{\varphi \in X ; u_{\bar{\varphi}}\right.$ has rapid decay $\}$. Moreover, $\mathscr{R}_{ \pm}$are unbounded subsets of $L^{2}\left(\mathbf{R}^{N}\right)$.

Proof. By Lemma 4.1, we need only show the result for $\mathscr{R}_{+}$. The first assertion follows from Propositions 3.15 and 3.14. Consider now $\varphi \in X$. For $b \geqq 0$, let $\varphi_{b}(x)=$ $e^{i\left(b|x|^{2}\right) / 4} \varphi(x)$. It follows from Corollary 2.5 that $\varphi_{b}(x) \in \mathscr{R}_{+}$, for $b$ large enough. Since $\left\|\varphi_{b}\right\|_{L^{2}}=\|\varphi\|_{L^{2}}$, it follows that $\mathscr{R}_{+}$is unbounded in $L^{2}\left(\mathbf{R}^{N}\right)$.

\section{Remarks}

As noted in the introduction, Corollary 2.5 shows that whether or not a solution to (1.1) blows up in finite time can not be determined solely from the modulus of the initial value $\varphi$. Indeed, appropriately modifying the phase of $\varphi$ produces a global, rapidly decaying solution. This suggests that blow up itself is a consequence of rotational properties of the solution, rather than the size of the modulus of the solution. 
We can make this idea more precise as follows. Consider $u \in C\left([0, T], H^{2}\left(\mathbf{R}^{N}\right)\right) \cap$ $C^{1}\left([0, T], L^{2}\left(\mathbf{R}^{N}\right)\right)$ with $u(0)=\varphi$. Set

$$
h(t)=\frac{\operatorname{Re} u \operatorname{Im} u_{t}-\operatorname{Re} u_{t} \operatorname{Im} u}{|u|^{2}} .
$$

It follows that

$$
\operatorname{Im} \bar{u} u_{t}=|u|^{2} h(t)
$$

and, if we write $u=\rho e^{i \theta}$, then $h(t)=\theta_{t}$. In other words, $h$ measures the speed of rotation of $u$.

Suppose first that $u$ is a solution of

$$
i u_{t}+\Delta u=0
$$

i.e. (1.1) with $\lambda=0$. Multiplying (5.3) by $\bar{u}$, integrating over $\mathbf{R}^{N}$, and taking the real part, we get that

$$
\int_{\mathbf{R}^{N}} \operatorname{Im} u_{t} \bar{u} d x+\|\nabla u\|_{L^{2}}^{2}=0 .
$$

In other words, since $\|\nabla u(t)\|_{L^{2}}^{2}=\|\nabla u(0)\|_{L^{2}}^{2}$ for solutions of $(5.3)$, we see that

$$
\int_{\mathbf{R}^{N}}|u(t)|^{2} h(t) d x=-\|\nabla \varphi\|_{L^{2}}^{2}
$$

Now suppose that $u$ is a solution of

$$
i u_{t}=-|u|^{\alpha} u
$$

i.e. (1.1) without the dispersion and with $\lambda<0$, which we take to be -1 . Multiplying (5.5) by $\bar{u}$ and taking the real part (without integrating) quickly yields that

$$
h(t)=|u(t)|^{\alpha} .
$$

The competition between the two parts of (1.1), i.e. Eqs. (5.3) and (5.5), is now evident. The linear equation (5.3) produces, on the average, a negative rotation, while the ordinary differential equation (5.5) produces a positive rotation at every point.

Finally, we suppose that $u$ is a solution of (1.1) with $\lambda=-1$ and $\alpha \geqq 4 / N$. Also, we suppose that $\varphi \in X$. Following the same steps as with Eq. (5.3) above, we arrive at the formula

$$
\int_{\mathbf{R}^{N}}|u(t)|^{2} h(t) d x=-2 E(\varphi)+\frac{\alpha}{\alpha+2}\|u(t)\|_{L^{x+2}}^{\alpha+2} .
$$

If $u$ is a global, rapidly decaying solution, then by Proposition $3.15,\|u(t)\|_{L^{x+2}}^{\alpha+2} \rightarrow 0$, as $t \rightarrow \infty$. Moreover, since $E(\varphi)<0$ implies finite time blow up (see $[11,24,4]$ ) and since $u$ being a rapidly decaying solution is an open condition on $\varphi$ (Theorems 4.2 and 4.12 above), we see that $E(\varphi)>0$. Thus,

$$
\int_{\mathbf{R}^{N}}|u(t)|^{2} h(t) d x \rightarrow-2 E(\varphi)<0,
$$

as $t \rightarrow \infty$. In this case, the negative rotation induced by (5.3) wins. If, on the other 
hand, $u$ blows up in finite time $T$, then $\|u(t)\|_{L^{\alpha+2}}^{\alpha+2} \rightarrow \infty$, as $t \uparrow T$; and so,

$$
\int_{\mathbf{R}^{N}}|u(t)|^{2} h(t) d x \rightarrow \infty
$$

as $t \uparrow T$. Here, the positive rotation induced by (5.6) wins. Furthermore, we can easily deduce the following information about $h(t)$ near blow up:

$$
\begin{gathered}
\|h(t)\|_{L^{\infty}} \underset{t \uparrow T}{\longrightarrow} \infty, \\
\int_{0}^{T}\|h(t)\|_{L^{(\alpha+2) / \alpha}}^{(\alpha+2) / \alpha}=\infty .
\end{gathered}
$$

The last identity follows easily from the property $\int_{0}^{T}\|u\|_{L^{\alpha+2}}^{\alpha+2}=\infty$ (see [5]).

Finally, we prove a result about global solutions to (1.1) in $X$ which are not rapidly decaying. If $\alpha<4 / N$, we know that such solutions are bounded in $H^{1}$. If $\alpha>4 / N$, we can at least show that a certain time average of the solution is bounded.

Proposition 5.1. Assume that $\alpha>4 / N$. Let $u \in C\left([0, \infty), H^{1}\left(\mathbf{R}^{N}\right)\right)$ be a global solution of (1.1), and assume that $u(0) \in X$. Then,

$$
\limsup _{t \rightarrow \infty} \frac{1}{t} \int_{0}^{t}\|\nabla u(s)\|_{L^{2}}^{2} d s \leqq \frac{4 N \alpha}{N \alpha-4} E,
$$

where $E=E(u(0))$.

Proof. With the notation of Remark 2.6, formula (2.11) can be written as

$$
f(t)=f(0)+t f^{\prime}(0)+2 N \alpha E(u(0)) t^{2}-2(N \alpha-4) \int_{0}^{t} \int_{0}^{s} \int_{\mathbf{R}^{N}}|\nabla u|^{2} d x d \sigma d s,
$$

from which it follows that

$$
2(N \alpha-4) \int_{0}^{t} \int_{0}^{s} \int_{\mathbf{R}^{N}}|\nabla u|^{2} d x d \sigma d s \leqq f(0)+t f^{\prime}(0)+2 N \alpha E(u(0)) t^{2}
$$

Note that

$$
\int_{0}^{t} \int_{0}^{s}\|\nabla u(\sigma)\|_{L^{2}}^{2} \geqq \int_{t / 2}^{t} \int_{0}^{t / 2}\|\nabla u(\sigma)\|_{L^{2}}^{2} \geqq t / 2 \int_{0}^{t / 2}\|\nabla u(s)\|_{L^{2}}^{2}
$$

Inequality (5.10) and the above inequality yield the result.

\section{References}

1. Barab, J. E.: Nonexistence of asymptotically free solutions for a nonlinear Schrödinger equation. J. Math. Phys. 25, 3270-3273 (1984)

2. Berestycki, H., Gallouet, T., Kavian, O.: Équations de champs scalaires Euclidiens nonlinéaires dans le plan. C. Rend. Acad. Sci. Paris 297, 307-310 (1983)

3. Berestycki, H., Lions, P.-L.: Nonlinear scalar field equations. Arch. Rat. Mech. Anal. 82, 313-375 (1983) 
4. Cazenave, T.: An introduction to nonlinear Schrödinger equations. Textos de Métodos Matemáticos vol. 22. I.M.U.F.R.J., Rio de Janeiro 1989

5. Cazenave, T., Weissler, F. B.: The Cauchy problem for the nonlinear Schrödinger equation in $H^{1}$. Manuscripta Math. 61, 477-494 (1988)

6. Cazenave, T., Weissler, F. B.: The Cauchy problem for the critical nonlinear Schrödinger equation in $H^{s}$. Nonlinear Anal, TMA 14, 807-836 (1990)

7. Cazenave, T., Weissler, F. B.: The structure of solutions to the pseudo conformally invariant nonlinear Schrödinger equation. Proc. Royal Soc. Edinburgh 117A, 251-273 (1991)

8. Constantin, P., Saut, J.-C.: Local smoothing properties of dispersive equations. J. Am. Math. Soc. 1, 413-439 (1988)

9. Constantin, P., Saut, J.-C.: Local smoothing properties of Schrödinger equations. Indiana Univ. Math. J. 38, 791-810 (1989)

10. Ginibre, J., Velo, G.: On a class of nonlinear Schrödinger equations. J. Funct. Anal. 32, 1-71 (1979)

11. Ginibre, J., Velo, G.: The global Cauchy problem for the nonlinear Schrödinger equation revisited. Ann. Inst. Henri Poincaré, Analyse Non Linéaire 2, 309-327 (1985)

12. Ginibre, J., Velo, G.: Scattering theory in the energy space for a class of nonlinear Schrödinger equations. J. Math. Pure Appl. 64, 363-401 (1985)

13. Ginibre, J., Velo, G.: Time decay of finite energy solutions of the nonlinear Klein-Gordon and Schrödinger equations. Ann. Inst. Henri Poincaré, Physique Théorique 43, 399-442 (1985)

14. Glassey, R. T.: On the asymptotic behavior of nonlinear wave equations. Trans. Am. Math. Soc. 182, 187-200 (1973)

15. Glassey, R. T.: On the blowing up of solutions to the Cauchy problem for nonlinear Schrödinger equations. J. Math. Phys. 18, 1794-1797 (1977)

16. Hayashi, N., Ozawa, T.: Lower bounds for order of decay or of growth in time for solutions to linear and nonlinear Schrödinger equations. Publ. Res. Inst. Math. Sci. (Kyoto Univ.) vol. 560. Kyoto Univ, 1986

17. Hayashi, N., Tsutsumi, Y.: Remarks on the scattering problem for nonlinear Schrödinger equations. In: Differential Equations and Mathematical Physics. Lecture Notes in Math. vol. 1285, pp. 162-168 Berlin, Heidelberg, New York: Springer 1987

18. Kato, T.: On nonlinear Schrödinger equations. Ann. Inst. Henri Poincaré, Physique Théorique 46, 113-129 (1987)

19. Keing, C., Ponce, G., Vega, L.: Oscillatory integrals and regularity of dispersive equations. Indiana Univ. Math. J. 40, 33-69 (1991)

20. Stein, E. M.: Singular integrals and differentiability of functions. Princeton, NJ: Princeton University Press, 1970

21. Strauss, W. A.: Nonlinear scattering theory. In: Scattering Theory in Mathematical Physics. pp. 53-78 (eds.). Lavita, J. A., Planchard, J.-P., Dordrecht: Reidel 1974

22. Strauss, W. A.: Nonlinear scattering theory at low energy. J. Funct. Anal. 41, 110-133 (1981)

23. Strauss, W. A.: Nonlinear wave equations. Regional Conference Series in Mathematics vol. 73, Providence, RI: Am. Math. Soc., 1989

24. Strauss, W. A.: The nonlinear Schrödinger equation. In: Contemporary developments in continuum mechanics and partial differential equations, pp. 452-465. De La Penha and Medeiros (eds.). Amsterdam: North-Holland, 1978

25. Strichartz, M.: Restrictions of Fourier transforms to quadratic surfaces and decay of solutions of wave equations. Duke Math. J. 44, 705-714 (1977)

26. Tsutsumi, Y.: Scattering problem for nonlinear Schrödinger equations. Ann. Inst. Henri Poincaré, Physique Théorique 43, 321-347 (1985)

27. Tsutsumi, Y., Yajima, K.: The asymptotic behavior of nonlinear Schrödinger equations. Bull. Am. Math. Soc. 11, 186-188 (1984)

28. Yajima, K.: Existence of solutions for Schrödinger evolution equations. Commun. Math. Phys. 110, 415-426 (1987)

29. Yajima, K.: The surfboard Schrödinger equations. Commun. Math. Phys. 96, 349-360(1984)

Communicated by H. Araki 